\title{
Property Rights Enforcement with Unverifiable Incomes*
}

\author{
Jan U. Auerbach ${ }^{\dagger}$
}

August 2, 2018

\begin{abstract}
I study the extent of secure property rights a planner can implement. Agents can produce output, appropriate others' output, or work in property rights enforcement. The planner pays enforcement personnel using taxes collected from producers who can hide income from taxation at a cost. The planner implements perfectly secure property rights by incentivizing production through redistributive taxation and absorbing potential appropriators as enforcement personnel. Both taxation and employment in enforcement institutionalize redistribution that would otherwise take place through appropriation. Higher costs of hiding income permit more redistributive taxation and less enforcement, leading to more production and higher welfare.
\end{abstract}

Keywords: Costly falsification, institutions, property rights, enforcement.

JEL classification: O17, P14.

*I thank Costas Azariadis for many discussions as well as three anonymous referees and participants at the Midwest Macro Meeting at Washington University in St. Louis, the International Meeting of the Association for Public Economic Theory at the University of Luxembourg, the Meeting of the European Public Choice Society at the University of Freiburg, the WINIR Symposium at the University of Bristol, the Midwest Economic Theory Conference at the University of Rochester, and the InsTED Workshop at Indiana University Bloomington. Errors are mine.

${ }^{\dagger}$ University of Exeter. E-mail: j.auerbach@exeter.ac.uk. Phone: +44 (0) 1392726115 . Postal address: University of Exeter Business School, 1.35 Streatham Court, Rennes Drive, Exeter EX4 4ST, United Kingdom. 


\section{Introduction}

Secure property rights matter for economic outcomes (see, e.g., Knack and Keefer 1995; Hall and Jones 1999; Acemoglu and Johnson 2005). A society looking to protect property rights needs to commit designated resources. The availability of means to hide income from taxation constrains society in its effort to collect these resources from its members. In the U.S., for example, the Internal Revenue Service estimates that, from 2008 to 2010, on average more than 16 percent of the total tax liability remained unpaid after enforced and late payments; and that tax underreporting accounted for 85 percent of the gap before enforced payments. ${ }^{1}$ Does this complication in raising funds affect society's choice of a property rights enforcement regime? Can it by itself help explain imperfectly secure property rights and differences across countries? That is, what could a planner achieve when agents can hide income from taxation if there were no other complications, like political economy frictions or agency problems?

I address these questions in an environment related to Murphy et al. (1993) and Acemoglu (1995). There are two types of agents with different productivities. Each agent can produce output, appropriate others' output, or work in enforcement. Those who produce output acquire property rights over it - the right to consume it, and the right to exclude others from consuming it. Protecting these property rights requires the planner to employ enforcement personnel to counter appropriation activities. In the context of this paper, appropriation activities are, for example, rent-seeking, corruption, theft and property crimes, fraud, or extortion; and they do not target particular groups nor require any special skill. ${ }^{2}$ In the first-best outcome both appropriation and enforcement are absent and property rights are perfectly secure. However, a planner can neither force agents into an occupation nor verify the true incomes of agents she wants to tax. Producers can misrepresent their income by hiding some of it at a cost so as to be subject to a different tax payment.

Although this informational friction constrains the planner, as soon as she can improve on anarchy, she does not tolerate any appropriation. She always achieves an outcome with perfectly secure property rights by employing enforcement personnel financed by redistributive taxation. This policy resembles the link between institutional investment and redistribution emphasized by Koeppl et al. (2014) for efficient contract enforcement in a production economy. ${ }^{3}$ Here, the planner may use subsidies to encourage unproductive agents to abstain from

\footnotetext{
${ }^{1}$ See Internal Revenue Service (2016). An estimate of the contribution of underreporting to the gap after enforced payments is not available. Tax compliance between 2008 and 2010 was comparable to that in 2006 (see pp. 6 and 8). See, e.g., Andreoni et al. (1998) on tax underpayment and evasion; see, e.g., Quintin (2008) on the link between limited contract enforcement, taxation, and informality.

${ }^{2}$ Tullock (1967), Rose-Ackerman (1975), and Becker (1968) started large literatures on some such activities. The availability of these activities affects the allocation of talent and resources. See, e.g., Baumol (1990), Murphy et al. (1991, 1993), Acemoglu (1995), and Acemoglu and Verdier (1998).

${ }^{3}$ On inequality, redistribution, and crime, see, e.g., Benoît and Osborne (1995), İmrohoroğlu et al. (2000).
} 
appropriation and instead contribute to the pie available to society. She uses the sector for enforcement to absorb all those who do not produce and might otherwise engage in appropriation. Taxation and employment in enforcement institutionalize redistribution, replacing appropriation. Higher costs of hiding income from taxation permit more income redistribution through taxation. A steeper tax schedule induces more agents to produce and allows the planner to employ fewer enforcement personnel. More agents producing more output increases aggregate consumption and welfare.

These results arise for three reasons. First, property rights are enforced by personnel whose wellbeing the planner values. Second, more taxation maps directly into more enforcement. While more taxation decreases the payoffs from both production and appropriation, more enforcement makes production more profitable relative to appropriation. At the same time, the planner pays enforcement personnel a fixed wage that needs to compensate them for their forgone outside option. In equilibrium, this outside option is appropriation and deteriorates with taxation and enforcement. It is thus always beneficial for the planner to tax producers slightly more and hire more enforcement personnel. Doing so absorbs potential appropriators and induces more agents to produce. The ability to subsidize unproductive producers strengthens this effect. Third, in equilibrium, enforcement personnel is recruited from a pool of agents that would otherwise engage in appropriation, not in production. Using unproductive rather than productive factors makes perfectly secure property rights affordable.

The literature explicitly linking taxation and property rights enforcement often focuses on state capacity for these two activities (e.g., Besley and Persson 2009, 2010; Herrera and Martinelli 2013) and on rulers or elites that use that capacity to serve their own interests (e.g., Olson 1993; Moselle and Polak 2001; Konrad and Skaperdas 2012; Acemoglu 2005). When considering taxation, important frictions arise from asymmetric information, which I model here along the lines of Lacker and Weinberg (1989). Grochulski (2007) and Casamatta (2011) study optimal taxation when taxes can be avoided by hiding income from taxation at a cost. While the environment here features a similar ex post moral hazard problem, it differs as agents can choose among three occupations which are directly affected or even established by the planner's choice of taxation and enforcement. The focus of this paper is also different: it is on the consequences of the informational friction for the security of property rights rather than for the optimal tax schedule. I do not address what determines the cost of hiding income from taxation. This endogenous economic institution is the subject of the literature on optimal income tax enforcement started by Reinganum and Wilde (1985), which connects to the literature on tax evasion started by Allingham and Sandmo (1972).

I present the model in Section 2, its predictions in Section 3, and a discussion in Section 4. 


\section{The Model}

There is a unit measure of risk neutral agents of two types and a benevolent planner. A measure $\mu_{l}>0$ of agents has low productivity $w_{l}>0$ and a measure $\mu_{h}>0$ of agents has high productivity $w_{h}>w_{l}$, where $\mu_{l}+\mu_{h}=1$. Individual productivities are private information and cannot be verified by the planner. Agents are endowed with one unit of time that is supplied indivisibly to one of three mutually exclusive occupations. An agent of type $i \in\{l, h\}$ can either produce $w_{i}$ units of the consumption good, work as enforcement personnel for a certain wage $w_{e}$, or engage in activities that may or may not lead to successful appropriation of a producer's income. Let $\chi_{i}^{j}$ be the share of agents of type $i \in\{l, h\}$ in occupation $j \in\{p, e, a\}$, where $p, e$, and $a$ indicate production, enforcement, and appropriation, respectively.

After production, producers of type $i$ display an income $z_{i}$ to the planner and pay a tax, or collect a subsidy, $t\left(z_{i}\right)$ that is indexed by and may vary with the income they display. ${ }^{4} \mathrm{~A}$ tax payment cannot exceed actual income. Producers of type $h$ can pretend to be of type $l$ by displaying $z_{h}=w_{l}$ so as to pay a different tax. To do so, they have to hide income $w_{h}-w_{l}$ at a cost captured by the function $\psi: \mathbb{R}_{+} \rightarrow \mathbb{R}_{+}$. While hiding nothing is costless, $\psi(0)=0$, the cost of hiding an amount $x>0$ is strictly positive, but less than $x$ : $0<\psi(x)<x$. Letting $\phi \equiv \psi\left(w_{h}-w_{l}\right)$, thus $\phi \in\left(0,\left(w_{h}-w_{l}\right)\right)$. All income not hidden is publicly observable. Producers can neither display more than their income nor hide all of it. ${ }^{5}$ The tax receipts are used to hire enforcers who are recognizable and safe from appropriation. They, and only they, can apprehend successful appropriators. Unsuccessful appropriators cannot be apprehended or punished in any way because they have not actually violated anyone's property rights.

Next, there are two rounds of random matching between agents. In the first round, appropriation takes place. Every agent can meet either an appropriator, a producer, or an enforcer. For any agent, the probability of meeting an agent of a certain type in a certain occupation is equal to the measure of agents of that type in that occupation. In particular, the probability $p$ of meeting an appropriator equals the share of appropriators in the population, $\chi_{l}^{a} \mu_{l}+\chi_{h}^{a} \mu_{h}$. Similarly, the probability $(1-\theta)$ of meeting an enforcer is $\chi_{l}^{e} \mu_{l}+\chi_{h}^{e} \mu_{h}$; the probability of meeting a producer of type $i$ is $\chi_{i}^{p} \mu_{i}$; the probability $q$ of meeting any producer at all is $\chi_{l}^{p} \mu_{l}+\chi_{h}^{p} \mu_{h}$. If a producer meets an appropriator, then the appropriator takes and runs off with all her resources, irrespective of whether or not they were hidden. That is, while producers can hide income from the planner, they cannot hide it from appropriators in the same way, because the latter do not face institutional or resource constraints, once they target someone's income. In all other meetings, no relevant interaction takes place: appropriators do not carry resources and enforcers are recognizable, preventing appropriation attempts.

\footnotetext{
${ }^{4}$ Following Lacker and Weinberg (1989), this assumption is without loss of generality (see Appendix A).

${ }^{5}$ At the solution of the planner's problem, agents of type $l$ do not want to display $w_{h}$, even if it is costless.
} 


\section{Table 1}

Timing

\begin{tabular}{|c|c|c|c|c|}
\hline 1. Regime Choice & 2. Production & 3. Enforcement & 4. Meetings & 5. Consumption \\
\hline $\begin{array}{l}\text { The planner } \\
\text { chooses and } \\
\text { enacts a regime. }\end{array}$ & $\begin{array}{l}\text { Agents choose } \\
\text { occupations and } \\
\text { producers produce. }\end{array}$ & $\begin{array}{l}\text { Taxes are collected } \\
\text { and enforcement } \\
\text { personnel is paid. }\end{array}$ & $\begin{array}{l}\text { Appropriation } \\
\text { and apprehension } \\
\text { take place. }\end{array}$ & $\begin{array}{l}\text { Agents consume } \\
\text { the resources they } \\
\text { have in hand. }\end{array}$ \\
\hline
\end{tabular}

In the second round, apprehension takes place. Successful appropriators - appropriators who met a producer in the first round - meet another agent. If a successful appropriator meets an enforcer, then the enforcer takes all the resources the appropriator carries and returns them to the producer who was expropriated of them. That is, the probability of apprehension equals the share of enforcement personnel in the population. The apprehended appropriator does not incur any additional costs besides zero consumption. In all other meetings, no relevant interaction takes place: successful appropriators cannot appropriate any more resources, cannot be expropriated by unsuccessful appropriators, and can be apprehended only by enforcers. After the second round of meetings, all agents consume the resources they carry.

The planner knows the productivity distribution captured by $\left(\mu_{l}, \mu_{h}, w_{l}, w_{h}\right)$ and the cost parameter $\phi$. At the outset, she chooses shares of agents of each type in each occupation, which also imply a measure of enforcement personnel to be employed, the tax schedule, and the wage paid to enforcers in order to maximize aggregate welfare with equal weights for all agents. After production, she collects taxes from producers, and pays enforcement personnel. She has to maintain a balanced budget: the expenses for wages paid to enforcement personnel have to equal the taxes collected, minus any subsidies. Table 1 summarizes the timing.

\section{Analysis}

I first specify the actions agents can take and the payoff functions these map into as well as the planner's objective function and problem to then state and discuss the implications of the model. All proofs can be found in Appendix C.

\subsection{Payoffs and the Planner's Problem}

A regime $\sigma \equiv\left(\chi_{l}^{p}, \chi_{l}^{e}, \chi_{l}^{a}, \chi_{h}^{p}, \chi_{h}^{e}, \chi_{h}^{a}, t_{l}, t_{h}, w_{e}\right) \in \Sigma \equiv[0,1]^{6} \times\left[-w_{h}, w_{l}\right] \times\left[-w_{h}, w_{h}\right] \times\left[0, w_{h}\right]$ collects the occupational assignments as captured by the shares of agents of each type in each occupation, the tax schedule, and the enforcement sector wage. All shares have to be nonnegative and cannot exceed one; taxes cannot exceed incomes $w_{l}$ and $w_{h}$, respectively; and a natural bound on subsidies and the nonnegative wages in enforcement is the income $w_{h}$ 
the most productive agents in the economy can generate. The planner takes into account the map from occupational choices (instructions) to the probabilities $q,(1-\theta)$, and $p$ of meeting a producer, an enforcer, or an appropriator, respectively, that is,

$$
q=\chi_{l}^{p} \mu_{l}+\chi_{h}^{p} \mu_{h} ; \quad(1-\theta)=\chi_{l}^{e} \mu_{l}+\chi_{h}^{e} \mu_{h} ; \quad p=\chi_{l}^{a} \mu_{l}+\chi_{h}^{a} \mu_{h} .
$$

After displaying income $z_{i}$ at the cost $\psi\left(w_{i}-z_{i}\right)$ and paying taxes $t\left(z_{i}\right)$, a producer with productivity $w_{i}$ carries resources $w_{i}-t\left(z_{i}\right)-\psi\left(w_{i}-z_{i}\right)$. Then, a producer meets an appropriator with probability $p$, in which case she is expropriated of all her resources. With probability $(1-\theta)$ an enforcer apprehends the appropriator and returns the resources; with probability $\theta$, the appropriator can run off with them. Therefore, the expected payoff an agent of type $i$ derives from production, when displaying income $z_{i}$ and paying both the designated tax $t\left(z_{i}\right)$ for producers displaying $z_{i}$ and the associated cost $\psi\left(w_{i}-z_{i}\right)$ of hiding income is given by

$$
[(1-\theta) p+(1-p)]\left(w_{i}-t\left(z_{i}\right)-\psi\left(w_{i}-z_{i}\right)\right)=(1-\theta p)\left(w_{i}-t\left(z_{i}\right)-\psi\left(w_{i}-z_{i}\right)\right) .
$$

When a producer chooses an income to display, she maximizes her payoff from doing so. However, the income display must be feasible and, given that the planner understands the incentives to falsify income, consistent with all agents' occupational choices: First, producers can neither display a higher income than they have generated nor hide all income. Second, if a producer were to display an income that, given the regime, no agent that produces generates, then the planner catches that and punishes this producer prohibitively high. That is, the only income display that is feasible for producers of type $l$ is $z_{l}=w_{l}$. The associated tax payment is $t\left(w_{l}\right)=t_{l}$. The expected payoff of a producer of type $l$ is given by the function $\varphi_{l}: \Sigma \rightarrow \mathbb{R}$,

$$
\varphi_{l}(\sigma)=(1-\theta p)\left(w_{l}-t_{l}\right)
$$

A producer of type $h$ can display $z_{h} \in\left\{w_{l}, w_{h}\right\}$, if some agents of type $l$ produce. If no agent of type $l$ produces, then the only income a producer of type $h$ can display that is consistent with $\sigma$ is $z_{h}=w_{h}$. The tax payment associated with the income display $w_{h}$ is $t\left(w_{h}\right)=t_{h}$. Thus, given any regime $\sigma \in \Sigma$, the income display $\zeta(\sigma)$ of a producer of type $h$ satisfies

$$
\zeta(\sigma) \in \arg \max _{z \in Z(\sigma)} w_{h}-t(z)-\psi\left(w_{h}-z\right)
$$

where

$$
Z(\sigma)= \begin{cases}\left\{w_{l}, w_{h}\right\} & \text { if } \chi_{l}^{p}>0 \\ \left\{w_{h}\right\} & \text { otherwise }\end{cases}
$$


is the set of all income displays $z_{h}$ that are consistent with the regime $\sigma$. To simplify notation, I suppress the dependence on the regime $\sigma$ and write $\zeta$ to mean $\zeta(\sigma)$ whenever there is no risk of confusion. At the optimal income display $\zeta$, the cost of hiding resources, $\psi\left(w_{h}-\zeta\right)$, is either zero if $\zeta=w_{h}$, or $\phi$ if $\zeta=w_{l}$. The expected payoff an agent of type $h$ can derive from producing and optimally displaying income is thus given by the function $\varphi_{h}: \Sigma \rightarrow \mathbb{R}$,

$$
\varphi_{h}(\sigma)=(1-\theta p)\left(w_{h}-t(\zeta)-\psi\left(w_{h}-\zeta\right)\right)=(1-\theta p) \max \left\{w_{h}-t_{h}, w_{h}-t_{l}-\phi\right\} .
$$

Given $\sigma$, only appropriators that meet a producer carry resources after the first round of meetings. In the second round of meetings, those successful appropriators escape apprehension with probability $\theta$. Only in this case do appropriators carry any resources after the meetings. Therefore, the expected payoff from appropriation is proportional to the expectation of a random draw from the set of producer incomes net of taxes and costs of hiding income. It is independent of the appropriator's productivity and thus the same for all appropriators. The expected payoff from appropriation can be written as a function $\nu: \Sigma \rightarrow \mathbb{R}$ given by

$$
\nu(\sigma)=\theta\left[\chi_{l}^{p} \mu_{l}\left(w_{l}-t_{l}\right)+\chi_{h}^{p} \mu_{h}\left(w_{h}-t(\zeta)-\psi\left(w_{h}-\zeta\right)\right)\right]
$$

Any agent that works as enforcement personnel receives a wage $w_{e}$ with certainty and her payoff from working in enforcement is thus $w_{e}$. The planner's balanced budget is given by

$$
\left(\chi_{l}^{e} \mu_{l}+\chi_{h}^{e} \mu_{h}\right) w_{e}=\chi_{l}^{p} \mu_{l} t_{l}+\chi_{h}^{p} \mu_{h} t(\zeta)
$$

implying that the tax receipts are nonnegative, because the wage bill of the enforcement sector is. The planner's objective function is aggregate welfare with equal weights for all agents:

$$
\chi_{l}^{p} \mu_{l} \varphi_{l}(\sigma)+\chi_{h}^{p} \mu_{h} \varphi_{h}(\sigma)+\left(\chi_{l}^{e} \mu_{l}+\chi_{h}^{e} \mu_{h}\right) w_{e}+\left(\chi_{l}^{a} \mu_{l}+\chi_{h}^{a} \mu_{h}\right) \nu(\sigma) .
$$

Using the payoff expressions (2)-(4), the balanced budget (5), and the definitions of the probabilities (1), which the planner understands, it can be written as (see Appendix B)

$$
\chi_{l}^{p} \mu_{l} w_{l}+\chi_{h}^{p} \mu_{h} w_{h}-\chi_{h}^{p} \mu_{h} \psi\left(w_{h}-\zeta\right) .
$$

That is, the planner's problem is to choose a regime $\sigma$ consisting of shares of agents of each type in each occupation, a tax schedule, and a wage in enforcement in order to maximize total production minus the cost of hiding all income that is being hidden. As she cannot dictate individual occupations, her choice of a regime needs to be incentive compatible in the sense that no agent should expect a higher payoff from switching occupations. In addition, the budget has to be balanced and the shares of agents of each type in each occupation have 
to add up to one. The planner's problem is

$$
\begin{array}{ll} 
& \max _{\sigma \in \Sigma} \quad \chi_{l}^{p} \mu_{l} w_{l}+\chi_{h}^{p} \mu_{h} w_{h}-\chi_{h}^{p} \mu_{h} \psi\left(w_{h}-\zeta\right) \\
\text { s.t. } & \left(\chi_{l}^{e} \mu_{l}+\chi_{h}^{e} \mu_{h}\right) w_{e}=\chi_{l}^{p} \mu_{l} t_{l}+\chi_{h}^{p} \mu_{h} t(\zeta) ; \\
& \varphi_{i}(\sigma) \geq \max \left\{w_{e}, \nu(\sigma)\right\} \quad \text { if } \chi_{i}^{p}>0, \quad \forall i ; \\
& w_{e} \geq \max \left\{\varphi_{i}(\sigma), \nu(\sigma)\right\} \quad \text { if } \chi_{i}^{e}>0, \quad \forall i ; \\
& \nu(\sigma) \geq \max \left\{\varphi_{i}(\sigma), w_{e}\right\} \quad \text { if } \chi_{i}^{a}>0, \quad \forall i ; \\
& \chi_{i}^{p}+\chi_{i}^{e}+\chi_{i}^{a}=1, \quad \forall i ; \\
& q=\chi_{l}^{p} \mu_{l}+\chi_{h}^{p} \mu_{h} ; \quad(1-\theta)=\chi_{l}^{e} \mu_{l}+\chi_{h}^{e} \mu_{h} ; \quad p=\chi_{l}^{a} \mu_{l}+\chi_{h}^{a} \mu_{h} .
\end{array}
$$

The objective function is expression (6). Constraint (7) requires the budget to be balanced. Constraint (8) says that a producer, that may choose to display a false income if she is of type $h$, should be at least as well off as she would be by taking up an activity in enforcement or appropriation. Similarly, constraints (9) and (10) require agents of either type that work in enforcement or appropriation to find it optimal to do so, instead of deviating to, for example, producing and hiding income in case they are of type $h$. Constraint (11) requires that the shares of agents of each type in each occupation add up to one. Constraint (12) states that the planner understands and takes into account how the shares of agents in all occupations map into the probabilities that enter the expected payoffs.

\subsection{Anarchy and The First-Best Outcome}

Before I study Problem (PP), in this section, I discuss two extreme scenarios: anarchy and the first-best outcome. First, suppose that there is neither a planner nor any other governing authority so that no taxes are collected and no enforcement personnel is hired, i.e., $\chi_{l}^{e}=$ $\chi_{h}^{e}=t_{l}=t_{h}=w_{e}=0$. In this case, in an equilibrium, all agents choose to engage in either production or appropriation in order to maximize their expected payoff, taking all other agents' occupational choices as given. I refer to this situation as anarchy.

Proposition 1. In anarchy, any share of agents of type $h$ produces, all others appropriate.

In anarchy, appropriation implements redistribution. If some agents of type $h$ produce output, then some redistribution takes place by appropriators taking resources from them after production. In particular, if all agents of type $h$ produce, then appropriation redistributes income to those who are not productive enough to choose to engage in production themselves. Without taxation and enforcement, redistribution can only occur through appropriation.

I next describe the first-best outcome and show that there is a regime with incentive compatible occupations that attains it. Suppose that the planner is only constrained by technology 
and resources - or agents' productivities and endowments with time. That is, besides choosing a tax schedule and the enforcement sector wage, she can dictate all agents' occupations, irrespective of whether or not agents agree with those occupation assignments, and verify producers' taxable incomes. The tax schedule can depend on actual income, rather than displayed income, and no costs from hiding income are incurred. The planner only has to balance the budget and ensure that all shares add up to one. Her problem is

$$
\begin{array}{ll} 
& \max _{\sigma \in \Sigma} \chi_{l}^{p} \mu_{l} w_{l}+\chi_{h}^{p} \mu_{h} w_{h} \\
\text { s.t. } & \left(\chi_{l}^{e} \mu_{l}+\chi_{h}^{e} \mu_{h}\right) w_{e}=\chi_{l}^{p} \mu_{l} t_{l}+\chi_{h}^{p} \mu_{h} t_{h} ; \\
& \chi_{i}^{p}+\chi_{i}^{e}+\chi_{i}^{a}=1, \quad \forall i .
\end{array}
$$

The following proposition characterizes the first-best outcome.

Proposition 2. The first-best regime has all agents produce, collects total tax receipts equal to zero, and implements no enforcement at all.

This first-best outcome derives from two aspects. First, both appropriation and enforcement are unproductive. Any agent that appropriates or enforces does not produce and thus not contribute to the pie the planner has available for distribution. Hence, the planner instructs all agents to produce so as to maximize output. Second, linear utility implies that the planner does not have an a priori incentive to redistribute resources among agents through transfers. Hence, any tax schedule that at most redistributes income through tax payments and subsidies is efficient - not implementing redistribution through the tax schedule at all thus is.

If the planner cannot dictate individual occupational choices, then she chooses incentive compatible occupations, the wage paid in enforcement, and the tax schedule, which can still depend on actual income. Her problem is

$$
\begin{array}{rll}
\left(\mathrm{FBP}^{\prime}\right) & \max _{\sigma \in \Sigma} \quad \chi_{l}^{p} \mu_{l} w_{l}+\chi_{h}^{p} \mu_{h} w_{h} \\
\text { s.t. } & \left(\chi_{l}^{e} \mu_{l}+\chi_{h}^{e} \mu_{h}\right) w_{e}=\chi_{l}^{p} \mu_{l} t_{l}+\chi_{h}^{p} \mu_{h} t_{h} ; \\
(13) \quad & (1-\theta p)\left(w_{i}-t_{i}\right) \geq \max \left\{w_{e}, \theta\left[\chi_{l}^{p} \mu_{l}\left(w_{l}-t_{l}\right)+\chi_{h}^{p} \mu_{h}\left(w_{h}-t_{h}\right)\right]\right\} \quad \text { if } \chi_{i}^{p}>0, \quad \forall i ; \\
(14) \quad & w_{e} \geq \max \left\{(1-\theta p)\left(w_{i}-t_{i}\right), \theta\left[\chi_{l}^{p} \mu_{l}\left(w_{l}-t_{l}\right)+\chi_{h}^{p} \mu_{h}\left(w_{h}-t_{h}\right)\right]\right\} \quad \text { if } \chi_{i}^{e}>0, \quad \forall i ; \\
(15) \quad & \theta\left[\chi_{l}^{p} \mu_{l}\left(w_{l}-t_{l}\right)+\chi_{h}^{p} \mu_{h}\left(w_{h}-t_{h}\right)\right] \geq \max \left\{(1-\theta p)\left(w_{i}-t_{i}\right), w_{e}\right\} \quad \text { if } \chi_{i}^{a}>0, \quad \forall i ; \\
& \chi_{i}^{p}+\chi_{i}^{e}+\chi_{i}^{a}=1, \quad \forall i ; \\
(16) \quad & q=\chi_{l}^{p} \mu_{l}+\chi_{h}^{p} \mu_{h} ; \quad(1-\theta)=\chi_{l}^{e} \mu_{l}+\chi_{h}^{e} \mu_{h} ; \quad p=\chi_{l}^{a} \mu_{l}+\chi_{h}^{a} \mu_{h} .
\end{array}
$$

The additional constraints (13)-(15) ensure that all agents can expect a payoff from their occupation - production, enforcement, or appropriation - that is at least as high as the maxi- 
mum payoff they could obtain from either one of the alternative occupations. Constraint (16) ensures that the occupational choices are consistent with the probabilities of meeting agents in certain occupations. The solution to Problem $\left(\mathrm{FBP}^{\prime}\right)$ implements the first-best outcome.

Proposition 3. There is a unique first-best regime with incentive compatible occupations. It has all agents produce, equalizes after-tax incomes, and implements no enforcement at all.

The planner instructs all agents to produce so as to maximize the pie. She then collects taxes and subsidizes unproductive agents with transfers paid for by productive agents. The transfers equalize consumption across agents so as to incentivize abstention from appropriation in favor of production. No resources are spent on enforcement at all. The intuition is unchanged: appropriation and enforcement do not contribute to the pie available to society.

\subsection{Unverifiable Incomes}

In this section, I study Problem (PP). A number of insights allow to simplify the problem. First, as is intuitive, the planner chooses to employ the most productive agents in production.

Lemma 1. All agents of type $h$ produce.

The planner can always achieve at least the best possible anarchy regime: a regime in which all agents of type $h$ produce, all agents of type $l$ appropriate, and no taxes are collected at all satisfies all constraints of Problem (PP) and attains a value of $\mu_{h} w_{h}$. Suppose that a strictly positive share of agents of type $l$ produces, and that those agents find it optimal to do so. Then, $\theta p<1$ and the after-tax income matters. As the cost $\phi$ of hiding income $\left(w_{h}-w_{l}\right)$ satisfies $\phi<\left(w_{h}-w_{l}\right)$, the after-tax income of producers of type $h$ is

$$
\max \left\{w_{h}-t_{h}, w_{h}-t_{l}-\phi\right\} \geq w_{h}-t_{l}-\phi>w_{h}-t_{l}-\left(w_{h}-w_{l}\right)=w_{l}-t_{l} .
$$

That is, the payoff an agent of type $h$ derives from production is strictly greater than that of an agent of type $l$. Hence, if agents of type $l$ at least weakly prefer production over the other two occupations, then all agents of type $h$ strictly prefer production over the other occupations. Individually optimal occupational choice then requires that all agents of type $h$ produce. That is, production of all agents of type $h$ is attainable; and if agents of type $l$ produce, then so do all agents of type $h$.

The only way for the planner to do better than anarchy is for the allocation to also have some agents of type $l$ produce. Any such regime departs from the first-best regime.

Lemma 2. Some enforcement is implemented if some agents of type l produce.

As indicated by inequality (17), the planner cannot equalize after-tax incomes, because producers of type $h$ can hide income from taxation. Thus, redistributive taxation alone cannot 
sufficiently counteract the incentive to engage in appropriation activities when other agents produce. The planner has to hire agents of type $l$ as enforcement personnel. As becomes clear in the proof of Lemma 2, if there was no enforcement at all, then these agents would choose to be appropriators. That is, enforcement personnel is hired from a pool of agents that would otherwise engage in appropriation. When raising taxes to pay for them, in order to prevent deadweight loss, the planner avoids tax schedules that induce income to be hidden.

Proposition 4. The tax schedule precludes hidden income if some agents of type l produce. This result resembles the no-falsification theorem proven by Grochulski (2007). For any regime that induces producers of type $h$ to hide income from taxation, there is an alternative regime in the constraint set, with a different tax schedule, that induces producers to display their true income and increases welfare. The resources that would be spent on hiding income in the original regime are collected as additional tax receipts that can be used to lower taxes on producers and pay higher wages to enforcers. As the shares of agents of each type in each occupation are the same in both regimes, they produce the same output. However, unlike in the original regime, in the alternative regime all output is consumed.

From Proposition 4 it thus follows that, if $\chi_{l}^{p}>0$, the regime induces truthful income displays, so that $\zeta=w_{h}, \psi\left(w_{h}-\zeta\right)=0$, and $t(\zeta)=t_{h}$. As all agents of type $h$ produce by Lemma 1 , implying that $\theta p<1$, it then follows from equation (3) that the tax schedule has to satisfy

$$
w_{h}-t_{h} \geq w_{h}-t_{l}-\phi
$$

Agents of type $h$ should not be willing to display income $w_{l}$ and incur both the associated tax payment $t_{l}$ and the cost $\phi$ of hiding output $\left(w_{h}-w_{l}\right)$. This requirement can be rewritten as

$$
t_{h} \leq t_{l}+\phi
$$

Inequality (19) reveals that the tax schedule cannot be too steep: the tax payment $t_{h}$ designated for a producer with high income $w_{h}$ cannot be too much higher than the one designated for a producer with lower income $w_{l}$. As one would expect, the upper bound on the difference between the two designated tax payments is determined by the cost of hiding income from taxation. Together, and as taxes cannot exceed incomes, inequalities (17) and (18) imply

$$
w_{h}-t_{h}>w_{l}-t_{l} \geq 0
$$

That is, more productive producers require a higher after-tax income than less productive producers. As $\chi_{l}^{p}>0$, it then follows from constraint (8) that agents of type $h$ strictly prefer production over the other two occupations, because $\varphi_{h}(\sigma)>\varphi_{l}(\sigma) \geq \max \left\{w_{e}, \nu(\sigma)\right\}$, which is consistent with $\chi_{h}^{p}=1$ and $\chi_{h}^{e}=\chi_{h}^{a}=0$. Therefore, constraints (8), (9), and (10) 
corresponding to agents of type $h$ can be ignored. For agents of type $l$, constraints (8), (9), and (10) imply indifference among all three occupations.

Lemma 3. Agents of type l are indifferent among occupations if some agents of type l produce.

If some agents of type $l$ produce, then some enforce, implying that constraints (8) and (9) have to hold for $i=l$. Combining them, agents of type $l$ should derive the same expected payoff from production and enforcement. If the share of agents of type $l$ in appropriation is strictly positive, then appropriation should provide the same expected payoff as well. However, the expected payoffs to agents of type $l$ from all three occupations have to be equal to each other even when no agent of type $l$ engages in appropriation activities. The reason is that, in order for them to abstain from appropriation in favor of another activity, agents of type $l$ need only be made at least as well off as if they engaged in appropriation. If production and enforcement promised a strictly greater expected payoff than appropriation, then the planner could afford to move some enforcement personnel into the production sector, thereby increasing output, adjusting taxes appropriately, without changing the relevant meeting probabilities enough to make appropriation profitable. That is, a regime that offers agents of type $l$ a strictly higher expected payoff in production and enforcement than it offers in appropriation is dominated by another regime in the constraint set that does not.

Combining the insights of Lemmas 1-3 and Proposition 4, if the candidate solution $\sigma$ has some agents of type $l$ produce, then it has to solve a simplified version of Problem (PP), focusing on regimes in the subset $\Sigma^{\prime} \equiv[0,1]^{3} \times\{1\} \times\{0\} \times\{0\} \times\left[-w_{h}, w_{l}\right] \times\left[-w_{h}, w_{h}\right] \times\left[0, w_{h}\right] \subset \Sigma$, with generic element $\sigma=\left(\chi_{l}^{p}, \chi_{l}^{e}, \chi_{l}^{a}, 1,0,0, t_{l}, t_{h}, w_{e}\right)$. The simplified planner's problem is

$$
\begin{array}{ll} 
& \max _{\sigma \in \Sigma^{\prime}} \quad \chi_{l}^{p} \\
\text { s.t. } & \chi_{l}^{e} \mu_{l} w_{e}=\chi_{l}^{p} \mu_{l} t_{l}+\mu_{h} t_{h} ; \\
& t_{l}+\phi \geq t_{h} ; \\
& \varphi_{l}(\sigma)=w_{e} ; \\
& w_{e}=\nu(\sigma) ; \\
& \chi_{l}^{p}+\chi_{l}^{e}+\chi_{l}^{a}=1 ; \\
& q=\chi_{l}^{p} \mu_{l}+\mu_{h} ; \quad(1-\theta)=\chi_{l}^{e} \mu_{l} ; \quad p=\chi_{l}^{a} \mu_{l} .
\end{array}
$$

As no income is hidden, the planner's objective function is aggregate output. It is maximized by maximizing the share of agents of type $l$ that join the agents of type $h$ in production. The constraint set of Problem ( $\left.\mathrm{PP}^{\prime}\right)$ is a subset of that of Problem (PP). By Lemmas 1-3 and Proposition 4, and the details of their proofs, for all regimes $\sigma$ with $\chi_{l}^{p}>0$ in the constraint set of Problem (PP), either $\sigma$ or another regime $\hat{\sigma}$ with $\hat{\chi}_{l}^{p}>0$ that is associated with a higher 
objective function value than $\sigma$ is in the constraint set of Problem $\left(\mathrm{PP}^{\prime}\right)$. Constraints (23) and (24) together state that an agent of type $l$ should expect the same payoff from all three occupations. Both appropriators and enforcers are agents of type $l$ and cannot pretend to have produced $w_{h}$, were they to deviate to production. As before, but accounting for the fact that all agents of type $h$ produce, constraint (21) requires a balanced budget; constraint (25) requires that the shares of agents of type $l$ in all occupations add up to one; and the equalities collected in constraint (26) state that the planner understands and takes into account how these shares map into the meeting probabilities. Constraint (22) indicates that the difference between the tax payments designated for producers of types $l$ and $h$ is bounded by the cost of hiding income from taxation. In fact, the planner exhausts this bound by setting taxes that make producers of type $h$ indifferent among income displays.

Lemma 4. Producers of type $h$ are indifferent among displays if some agents of type l produce.

The planner implements a tax schedule such that $t_{h}=t_{l}+\phi$. Redistributive taxation is one means of inducing rather unproductive agents to abstain from appropriation and engage in production instead. Unlike enforcement personnel, who are paid from the tax receipts collected, agents of type $l$ that redistribution induces to produce do contribute to the pie available to society. The planner's regime exhausts the bound on the difference between the taxes facing productive and unproductive producers to exploit the advantages of redistributive taxation as much as possible. This aspect plays an important role in discarding appropriation.

Proposition 5. The allocation precludes appropriation if some agents of type l produce.

If a regime in which some agents of type $l$ produce also has some agents of type $l$ engage in appropriation, then the planner can reallocate some of those appropriators to production, and reassign the rest of them to the enforcement sector. As a result property rights are perfectly secure, which would compensate producers for higher taxes, should financing the wage bill of the grown enforcement sector command them. While more production tends to increase the expected payoff from appropriation, the higher number of enforcement personnel and, if required, the higher taxes paid by producers tend to decrease it. As a consequence, high enough wages in enforcement to compensate would-be appropriators are affordable.

Combining the above insights, the solution to the planner's problem can be fully characterized.

Proposition 6. Problem (PP) has a solution. If $\phi \leq \underline{\phi} \equiv\left(\mu_{h} w_{h}-\left(1+\mu_{l} \mu_{h}\right) w_{l}\right) /\left(1+\mu_{l} \mu_{h}\right)$, then the planner cannot improve on the best anarchy regime: all agents of type $h$ produce, and only those. If $\phi>\underline{\phi}$, then there is a unique solution: all agents of type $h$ and some agents of type $l$ produce, all agents that do not produce are employed in enforcement. If the costs of hiding income from taxation are high enough, then producers of type l receive a subsidy. 
For some parameter constellations, the planner cannot improve on the best anarchy regime if the cost of hiding income from taxation is too small. In this case, the planner's capacity to raise taxes is constrained enough to prevent her from financing enough redistribution and enforcement. The planner can improve on anarchy if the cost of hiding income satisfies $\phi>\underline{\phi} .{ }^{6}$ Using $\mu_{l}=1-\mu_{h}$, the lower bound $\phi$ increases with (i) increases in the measure $\mu_{h}$ of agents of type $h$, all of whom will be producers; (ii) increases in the productivity $w_{h}$ of producers of type $h$; (iii) decreases in the productivity $w_{l}$ of producers of type $l$; and (iv) increases in inequality in the sense of a mean-preserving spread with the shares of agents in the population held constant. More producers of type $h$, more productive producers of type $h$, less productive producers of type $l$, and more inequality all increase the incentive for agents of type $l$ to engage in appropriation. More redistribution and enforcement are required to counteract this increased incentive. Thus, in order to improve on anarchy, the planner needs to raise more taxes, and hence requires more capacity for taxation, as measured by higher costs of hiding income from it. I discuss parameter constellations that are consistent with $\phi>\underline{\phi}$ in Section 4 below. To the extent that a society does not seem to be in anarchy, $\phi>\underline{\phi}$ does represent the relevant subset of the parameter space. The planner's solution then improves on anarchy, and absent any other frictions it provides an upper bound on this society's outcome.

If the planner can improve on anarchy, then she employs enough enforcement personnel so as to crowd out appropriation altogether, and property rights are perfectly secure. She does so by absorbing potential appropriators into the sector for enforcement for a certain wage. Appropriation is never attempted. Enforcement personnel does not actually provide a service other than being present-and thus effectively deterring appropriation efforts. Being employed in enforcement largely amounts to refraining from appropriation. The wage paid in enforcement amounts to a transfer financed by taxing producers. Employment in enforcement thus institutionalizes redistribution that would otherwise take place through appropriation.

While taxes may distort the occupational choice between production and appropriation, higher tax payments decrease the expected payoffs from both activities. This effect is present in general, independent of the exact tax schedule in place, and as long as utility is increasing. Thus, taxation affects the choice between either one of those two occupations and employment in enforcement. Too high taxes may draw productive agents (as well as appropriators) into the enforcement sector. The threat of expropriation of the resources an agent carries, however, affects the occupational choice between production and appropriation - as does the probability of apprehension. Intermediated by the sector for enforcement, taxation increases the probability of productive agents being able to reap the returns to their productive activity, which increases the incentive to produce. At the same time, the incentive to engage in appro-

\footnotetext{
${ }^{6}$ There is a $\underline{\underline{\phi}}<\underline{\phi}$ so that if $\phi \in[\underline{\underline{\phi}} \underline{\underline{\phi}}]$, no feasible regime improves on anarchy, but some avoid appropriation.
} 
priation activities decreases because the probability of escaping apprehension after successful appropriation decreases. That is, more agents prefer to produce. This effect, too, is present in general, independent of the exact tax schedule in place, and as long as utility is increasing. In addition, unproductive agents lean more towards switching to an occupation in the enforcement sector. Thus, the planner optimally employs rather unproductive members of society that do not produce as enforcement personnel at a wage that makes them indifferent between enforcement and appropriation. As she absorbs all potential appropriators into the enforcement sector, there is no appropriation and property rights are perfectly secure.

This security of property rights derives from the availability of a sector for enforcement of those rights because it provides an alternative occupation. That occupation is unproductive, as is appropriation. However, in contrast to appropriation, it does not harm the incentives of more productive agents to actually produce. Perfectly secure property rights are not too costly because enforcement personnel is recruited from a pool of agents that would otherwise engage in appropriation. While enforcers do not produce, enforcement does not withdraw agents from productive activities. Moreover, the resources spent on enforcement finance the consumption of enforcement personnel, which the planner values.

The tax profile that finances enforcement is redistributive. High income agents pay higher taxes than - and may even finance transfers to - low income agents. In fact, the tax facing producers of type $l$ is negative if the cost of hiding income is high enough. Negative taxes for unproductive producers subsidize their production, which contributes to the pie society has available for distribution amongst its members. These subsidies also incentivize abstention from appropriation, which would hurt others' incentives to produce. Redistributive taxation thus plays an important role, and it is easier to implement it in societies in which it is more costly for agents to hide income from taxation.

Proposition 7. Let $\phi>\underline{\phi}$. Societies with higher costs of hiding income from taxation enact a steeper tax profile with a smaller minimum tax, employ fewer enforcement personnel at a higher wage, see more productive activity, produce more output, and experience higher welfare.

That is, if the costs of hiding income from taxation are higher, then the planner can implement more redistribution through taxation. She can and does then choose to employ fewer agents in enforcement and to enact a steeper tax profile, with a larger difference between the tax payments facing productive and unproductive agents. That steeper tax profile-possibly offering (larger) subsidies to unproductive producers - encourages some agents to produce that would choose to enforce if the tax schedule were to implement less redistribution. As a consequence, fewer agents work in enforcement and more agents produce more output that can be shared among the members of society, which increases welfare. As aggregate consumption rises, agents employed in enforcement share in that rise via higher wages. 


\section{Discussion}

In this section, I briefly discuss a number of assumptions. For example, I assume that the planner maximizes aggregate welfare with equal weights for all agents. I take the view that there is no a priori reason to exclude appropriators' welfare from the planner's considerations - or assign a smaller weight to it. Appropriation is a form of redistribution; and the planner's solution institutionalizes another one, using taxation and enforcement. The assumption of linear utility ensures that the planner does not have an inherent desire to redistribute income. All redistribution the planner implements is thus driven by the need to provide agents with incentives. Strictly concave utility would intensify the planner's desire to both redistribute incomes across agents and reduce the uncertainty implied by insecure property rights.

I assume that the probability of any agent meeting another agent in a certain occupation equals the measure of agents in that occupation. In particular, for any agent, the probability of meeting an appropriator equals the measure of appropriators, i.e., the share of appropriators in the population. Acemoglu (1995) and İmrohoroğlu et al. (2000), among others, use a similar specification. While it simplifies the analysis, it shares qualitative features with other specifications, such as a constant returns to scale matching function: all else equal, more appropriators and fewer producers increase the probability of meeting an appropriator. In fact, this specification is unfriendly towards the results. The assumption that the probability of apprehension equals the share of enforcement personnel in the population makes secure property rights more costly than others. Among all concave (production) functions mapping the share of enforcement personnel in the population into a probability of apprehension, with zero and one mapping into zero and one, respectively, a linear function gives a lower probability than any strictly concave function everywhere in the interior of the domain. Similarly, successful appropriators that are apprehended by enforcement personnel are not subject to any punishment other than zero consumption (see, e.g., Becker 1968; Stigler 1970; Ehrlich 1973, and many others). Here, imposing a potentially costly punishment might make it easier to induce abstention from appropriation. However, the regime the planner chooses in the present environment remains attainable in that case.

One could assume that enforcement personnel may explicitly divert the resources recovered from apprehended appropriators without changing the results. The solution to the planner's problem I analyze would still be attainable, dominate all other regimes, and leave no room for such a deviation as there is no appropriation in the first place. In fact, the possibility of corrupt enforcement personnel grabbing resources themselves is captured to the extend that appropriation activities are a stylized description of unproductive redistribution of resources, including corruption. Moreover, in order to focus on a planner facing the friction that income can be hidden from taxation, I do not consider corruptible tax collectors (see, e.g., Chander 
and Wilde 1992; Besley and McLaren 1993; Hindriks et al. 1999). I thus abstract from an analysis of government agents' incentives and their implications altogether (see, e.g., Becker and Stigler 1974; Basu et al. 1992; Mookherjee and Png 1995; Acemoglu and Verdier 1998, 2000; Polinsky and Shavell 2001). The lack of a choice of effort to exert when an agent is employed in enforcement seems to be less relevant than the possibility of diverting recovered resources. Given the latter option, enforcement personnel would want to ensure a high probability of apprehending successful appropriators.

Proposition 6 distinguishes between two cases and states that for the planner to be able to improve on anarchy, the condition $\phi>\underline{\phi}=\left(\mu_{h} w_{h}-\left(1+\mu_{l} \mu_{h}\right) w_{l}\right) /\left(1+\mu_{l} \mu_{h}\right)$ must hold. A sufficient condition for this requirement to be satisfied for all $\phi>0$ is that $\underline{\phi} \leq 0$, or $\left(1+\mu_{l} \mu_{h}\right) w_{l} \geq \mu_{h} w_{h}$. This inequality can be rewritten as $\left(1-\mu_{h}^{2}\right) w_{l}=\left(1-\mu_{h}\left(1-\mu_{l}\right)\right) w_{l} \geq$ $\mu_{h}\left(w_{h}-w_{l}\right)$. A sufficient condition for it to hold therefore is that the income that all agents of type $l$ could generate is at least as high as the potential hidden income of all agents of type $h$ : $\mu_{l} w_{l} \geq \mu_{h}\left(w_{h}-w_{l}\right)$. If one is willing to think of the U.S. as a benchmark represented by the first-best outcome in the rather stylized environment here, then this condition in principle allows the parameters to qualitatively capture at least some aspects of the U.S. income distribution. For example, according to some measures, from 1917 to 2014 the income share of the top 10 percent has always been less than 50 percent (Piketty et al. 2018). That is, the income share of the lower 90 percent was larger than that of the top 10 percent. Letting $\mu_{h}=0.1$ and $\mu_{l}=0.9$, the income share of the top 10 percent in the first-best is $\mu_{h} w_{h} /\left(\mu_{l} w_{l}+\mu_{h} w_{h}\right)$, while that of the lower 90 percent is $\mu_{l} w_{l} /\left(\mu_{l} w_{l}+\mu_{h} w_{h}\right)$. Any $w_{l}>0$ and $w_{h}>w_{l}$ such that $\mu_{l} w_{l} \geq \mu_{h} w_{h}$ would be qualitatively consistent with this data and satisfy the parameter restriction $\mu_{l} w_{l} \geq \mu_{h}\left(w_{h}-w_{l}\right)$. Clearly, imposing $\mu_{l} w_{l} \geq \mu_{h} w_{h}$, and thus looking at the top 10 percent versus the lower 90 percent, is far from necessary to satisfy the condition $\mu_{l} w_{l} \geq \mu_{h}\left(w_{h}-w_{l}\right)$, which itself is sufficient for $\phi>0 \geq \underline{\phi}$ to hold. Similarly, not requiring $\underline{\phi} \leq 0$ allows to further relax restrictions on the productivity distribution.

According to the U.S. Internal Revenue Service (2016), improved visibility in the sense of information reporting and tax withholding improves tax compliance. Andreoni et al. (1998) argue that it is more difficult to hide income from taxation in developed countries than it is to do the same in developing ones, and that income derived from farms or sole proprietorship is particularly prone to evasion (p. 821). In addition, Schneider and Enste (2000) point to a role for the informal sector in tax evasion and provide estimates of its size to show that it is more prevalent in developing and transition economies than in developed countries. Therefore, while the analysis here abstracts from many important dimensions along which countries at different stages of development differ, a possible interpretation of the level of costs of hiding income from taxation might be as follows. One could imagine a society with a well governed and equipped tax authority; with a well developed financial market in which 
participants have effective screening devices available, and many companies are required to regularly report to, and are subject to audits by agents of, other participants; with a relatively important heavy and manufacturing industry, and a relatively less important agrarian sector; and with relatively many big enough companies that require a well established organizational form with cross-checks and within-firm bureaucracy to operate effectively. One could also imagine another society with an understaffed tax authority; with a large informal sector; with a relatively important agrarian sector; with large rural areas that are far behind the urban centers, both technologically and administratively; in which most firms are small enterprises, with a single owner often being the single employee. One might expect that it would be more difficult and thus costly to hide income and escape reporting duties in the former society.

\section{Conclusion}

In a model of appropriation and endogenous enforcement of property rights, I analyzed what a planner can achieve when she is unable to verify taxable incomes. I find that this friction cannot by itself help explain imperfectly secure property rights. Although it induces a binding and effective constraint on the planner's regime choice, as soon as she can improve on anarchy, it does not prevent her from implementing perfectly secure property rights. To do so, she uses a mix of redistributive taxation and employment of potential appropriators in enforcement. When higher costs of hiding income from taxation allow for more redistribution through taxation, the planner chooses to absorb fewer agents in enforcement and subsidize more unproductive producers.

\section{Appendices}

\section{A Tax Schedules}

I briefly replicate the argument given in Lacker and Weinberg (1989) for this environment. A mechanism consists of a message space $M$ and a tax schedule $t$ that maps the message and the income displayed into $\mathbb{R}$. A producer $w$ chooses a message $m(w) \in M$ and an income display $z(w)$ to maximize $w-t(m(w), z(w))-\psi(w-z(w))$. Suppose two agents with different productivities $w_{1}$ and $w_{2} \neq w_{1}$ were to send different messages $m\left(w_{1}\right)=m_{1}$ and $m\left(w_{2}\right)=$ $m_{2} \neq m_{1}$ but display the same income $z\left(w_{1}\right)=z\left(w_{2}\right)=\hat{z}$. By optimality of message and display, for agent $w_{1}, t\left(m_{1}, \hat{z}\right) \leq t\left(m_{2}, \hat{z}\right)$, while for agent $w_{2}, t\left(m_{1}, \hat{z}\right) \geq t\left(m_{2}, \hat{z}\right)$, so that

$t\left(m_{1}, \hat{z}\right)=t\left(m_{2}, \hat{z}\right)$. The same income display implies the same tax payment, irrespective of the message, which justifies focusing on tax schedules that only depend on displayed income. 


\section{B The Planner's Objective Function}

Using the payoff expressions (2)-(4), the balanced budget (5), and the definitions of the probabilities (1), which the planner understands, the planner's objective function is given by

$$
\begin{aligned}
& \chi_{l}^{p} \mu_{l} \varphi_{l}(\sigma)+\chi_{h}^{p} \mu_{h} \varphi_{h}(\sigma)+\left(\chi_{l}^{e} \mu_{l}+\chi_{h}^{e} \mu_{h}\right) w_{e}+\left(\chi_{l}^{a} \mu_{l}+\chi_{h}^{a} \mu_{h}\right) \nu(\sigma) \\
= & (1-\theta p)\left[\chi_{l}^{p} \mu_{l}\left(w_{l}-t_{l}\right)+\chi_{h}^{p} \mu_{h}\left(w_{h}-t(\zeta)\right)\right]-(1-\theta p) \chi_{h}^{p} \mu_{h} \psi\left(w_{h}-\zeta\right) \\
& +\left[\chi_{l}^{p} \mu_{l} t_{l}+\chi_{h}^{p} \mu_{h} t(\zeta)\right]+\theta p\left[\chi_{l}^{p} \mu_{l}\left(w_{l}-t_{l}\right)+\chi_{h}^{p} \mu_{h}\left(w_{h}-t(\zeta)\right)\right]-\theta p \chi_{h}^{p} \mu_{h} \psi\left(w_{h}-\zeta\right) \\
= & \chi_{l}^{p} \mu_{l}\left(w_{l}-t_{l}\right)+\chi_{h}^{p} \mu_{h}\left(w_{h}-t(\zeta)\right)-\chi_{h}^{p} \mu_{h} \psi\left(w_{h}-\zeta\right)+\chi_{l}^{p} \mu_{l} t_{l}+\chi_{h}^{p} \mu_{h} t(\zeta) \\
= & \chi_{l}^{p} \mu_{l} w_{l}+\chi_{h}^{p} \mu_{h} w_{h}-\chi_{h}^{p} \mu_{h} \psi\left(w_{h}-\zeta\right) .
\end{aligned}
$$

\section{Proofs}

\section{Proposition 1}

Proof. In anarchy, $\sigma=\left(\chi_{l}^{p}, 0, \chi_{l}^{a}, \chi_{h}^{p}, 0, \chi_{h}^{a}, 0,0,0\right), \varphi_{i}(\sigma)=(1-p) w_{i}, i=l, h$, and $\nu(\sigma)=$ $\left(\chi_{l}^{p} \mu_{l} w_{l}+\chi_{h}^{p} \mu_{h} w_{h}\right)$, where $p=\chi_{l}^{a} \mu_{l}+\chi_{h}^{a} \mu_{h}$ and $\chi_{i}^{a}=1-\chi_{i}^{p}, i=l, h$. First, in equilibrium, $\chi_{l}^{p}=0$. Suppose for a contradiction that $\chi_{l}^{p}>0$. Then, it must hold that $\varphi_{h}(\sigma)>\varphi_{l}(\sigma) \geq$ $\nu(\sigma)$ so that all agents of type $h$ produce, $\chi_{h}^{p}=1$. It follows that $\nu(\sigma)=\left(\chi_{l}^{p} \mu_{l} w_{l}+\mu_{h} w_{h}\right)>$ $\left(\chi_{l}^{p} \mu_{l}+\mu_{h}\right) w_{l}=(1-p) w_{l}=\varphi_{l}(\sigma)$, a contradiction. Thus, $\chi_{l}^{p}=0$ and $\chi_{l}^{a}=1$. For any $\chi_{h}^{p} \in[0,1], \varphi_{h}(\sigma)=(1-p) w_{h}=\left(1-\mu_{l}-\chi_{h}^{a} \mu_{h}\right) w_{h}=\left(\mu_{h}-\chi_{h}^{a} \mu_{h}\right) w_{h}=\left(1-\chi_{h}^{a}\right) \mu_{h} w_{h}=$ $\chi_{h}^{p} \mu_{h} w_{h}=\nu(\sigma) \geq \chi_{h}^{p} \mu_{h} w_{l}=(1-p) w_{l}$. That is, any profile of occupational choices that has any share of agents of type $h$ producing and all other agents appropriating maximizes all agents' expected payoffs, given all others' occupational choices, and thus is an equilibrium.

\section{Proposition 2}

Proof. The objective function in Problem (FBP) is less than or equal to $\bar{w}=\mu_{l} w_{l}+\mu_{h} w_{h}$ and equals $\bar{w}$ if and only if $\chi_{l}^{p}=\chi_{h}^{p}=1$ so that $\chi_{l}^{e}=\chi_{h}^{e}=0$, implying $\chi_{l}^{p} \mu_{l} t_{l}+\chi_{h}^{p} \mu_{h} t_{h}=0$.

\section{Proposition 3}

Proof. The objective function in Problem $\left(\mathrm{FBP}^{\prime}\right)$ is less than or equal to $\bar{w}=\mu_{l} w_{l}+\mu_{h} w_{h}$ and equals $\bar{w}$ if and only if $\chi_{l}^{p}=\chi_{h}^{p}=1$ so that $\chi_{l}^{e}=\chi_{h}^{e}=0$, implying $\chi_{l}^{p} \mu_{l} t_{l}+\chi_{h}^{p} \mu_{h} t_{h}=0$. Consider the regime given by $\chi_{l}^{p}=\chi_{h}^{p}=1, \chi_{l}^{e}=\chi_{h}^{e}=\chi_{l}^{a}=\chi_{h}^{a}=0, t_{l}=w_{l}-\bar{w}, t_{h}=w_{h}-\bar{w}$, and $w_{e}=0$. The tax receipts equal $\mu_{l}\left(w_{l}-\bar{w}\right)+\mu_{h}\left(w_{h}-\bar{w}\right)=\mu_{l} w_{l}+\mu_{h} w_{h}-\left(\mu_{l}+\mu_{h}\right) \bar{w}=$ $\bar{w}-\bar{w}=0$. As $p=0,(1-\theta p)\left(w_{i}-t_{i}\right)=\bar{w}$; as $\theta=1, \theta\left[\chi_{l}^{p} \mu_{l}\left(w_{l}-t_{l}\right)+\chi_{h}^{p} \mu_{h}\left(w_{h}-t_{h}\right)\right]=\bar{w}$. Thus, this regime satisfies all constraints of Problem $\left(\mathrm{FBP}^{\prime}\right)$ and implements the first-best. It is the only regime that does so: if $w_{l}-t_{l} \neq w_{h}-t_{h}$, then constraint (13) is violated for one 
of the two types of agents as $\theta\left[\chi_{l}^{p} \mu_{l}\left(w_{l}-t_{l}\right)+\chi_{h}^{p} \mu_{h}\left(w_{h}-t_{h}\right)\right]=\mu_{l}\left(w_{l}-t_{l}\right)+\mu_{h}\left(w_{h}-t_{h}\right)>$ $\min \left\{w_{l}-t_{l}, w_{h}-t_{h}\right\}$; if $w_{l}-t_{l}=w_{h}-t_{h}$, then the balanced budget, together with zero tax receipts after transfers, implies that $w_{l}-t_{l}=w_{h}-t_{h}=\bar{w}$.

\section{Lemma 1}

Proof. First, production by all agents of type $h$ can be attained. Consider the regime $\sigma$ that has all agents of type $h$ produce and is given by $\chi_{h}^{p}=\chi_{l}^{a}=1, \chi_{h}^{e}=\chi_{h}^{a}=\chi_{l}^{p}=\chi_{l}^{e}=0, t_{l}=t_{h}=0$, $w_{e}=0$. Then, $\theta=1$ and $p=\mu_{l}$ so that $\varphi_{h}(\sigma)=\left(1-\mu_{l}\right) w_{h}=\mu_{h} w_{h}, \varphi_{l}(\sigma)=\left(1-\mu_{l}\right) w_{l}=$ $\mu_{h} w_{l}$, and $\nu(\sigma)=\mu_{h} w_{h}$. Therefore, $\varphi_{h}(\sigma) \geq \max \left\{w_{e}, \nu(\sigma)\right\}$ and $\nu(\sigma) \geq \max \left\{\varphi_{l}(\sigma), w_{e}\right\}$. Thus, $\sigma$ satisfies all constraints of Problem (PP) and is thus attainable.

Second, if any agents of type $l$ produce, then all agents of type $h$ produce. Consider a candidate regime $\sigma$ and suppose for a contradiction that $\chi_{l}^{p}>0$ and $\chi_{h}^{p}<1$. Then, $\theta p<1$ so that $\varphi_{h}(\sigma)=(1-\theta p) \max \left\{w_{h}-t_{h}, w_{h}-t_{l}-\phi\right\} \geq(1-\theta p)\left(w_{h}-t_{l}-\phi\right)>(1-\theta p)\left(w_{h}-t_{l}-\right.$ $\left.\left(w_{h}-w_{l}\right)\right)=(1-\theta p)\left(w_{l}-t_{l}\right)=\varphi_{l}(\sigma)$, because $w_{h}-w_{l}>\phi$. But, as $\chi_{l}^{p}>0$, constraint (8) requires that $\varphi_{l}(\sigma) \geq \max \left\{w_{e}, \nu(\sigma)\right\}$. That is, $\varphi_{h}(\sigma)>\max \left\{w_{e}, \nu(\sigma)\right\}$, which violates at least one of the constraints (9) and (10), as $\chi_{h}^{p}<1$ implies via constraint (11) that either $\chi_{h}^{e}>0$, or $\chi_{h}^{a}>0$, or both. Thus, $\sigma$ is not in the constraint set of Problem (PP), a contradiction that completes the proof.

\section{Lemma 2}

Proof. Consider a candidate regime $\sigma$ and suppose for a contradiction that $\chi_{l}^{p}>0$ and $\chi_{l}^{e}=0$. As $\chi_{h}^{p}=1$ by Lemma $1, \chi_{h}^{e}=\chi_{l}^{e}=0$ and thus $\theta=1$. Therefore, $p=1-q$ so that $\varphi_{l}(\sigma)=(1-p)\left(w_{l}-t_{l}\right)=q\left(w_{l}-t_{l}\right)$. At the same time, $\nu(\sigma)=\chi_{l}^{p} \mu_{l}\left(w_{l}-t_{l}\right)+\mu_{h} \max \left\{w_{h}-\right.$ $\left.t_{h}, w_{h}-t_{l}-\phi\right\} \geq \chi_{l}^{p} \mu_{l}\left(w_{l}-t_{l}\right)+\mu_{h}\left(w_{h}-t_{l}-\phi\right)>\chi_{l}^{p} \mu_{l}\left(w_{l}-t_{l}\right)+\mu_{h}\left(w_{h}-t_{l}-\left(w_{h}-w_{l}\right)\right)=$ $\chi_{l}^{p} \mu_{l}\left(w_{l}-t_{l}\right)+\mu_{h}\left(w_{l}-t_{l}\right)=q\left(w_{l}-t_{l}\right)$, because $w_{h}-w_{l}>\phi$. That is, $\nu(\sigma)>\varphi_{l}(\sigma)$, which violates constraint (8), because $\chi_{l}^{p}>0$. Thus, $\sigma$ is not in the constraint set of Problem (PP), a contradiction that completes the proof.

\section{Proposition 4}

Proof. Consider any regime $\sigma$ with $\chi_{h}^{p}=1, \chi_{l}^{p}>0$, and, by Lemma 2, $\chi_{l}^{e}>0$ that satisfies all constraints of Problem (PP) and induces producers of type $h$ to hide income from taxation. That is, $w_{h}-t_{l}-\phi>w_{h}-t_{h}$. The value of the objective function is $\chi_{l}^{p} \mu_{l} w_{l}+\mu_{h} w_{h}-\mu_{h} \phi$. I show that there is an alternative regime $\hat{\sigma}$ in the constraint set which has the same occupation assignments but a different tax schedule that prevents income from being hidden and so increases welfare, and thus dominates $\sigma$. There are two cases: $\chi_{l}^{a}=0$ and $\chi_{l}^{a}>0$.

First, suppose $\chi_{l}^{a}=0$. Then, $p=0,(1-\theta)=\chi_{l}^{e} \mu_{l}=1-q$, and from constraints (8) and (9) 
for agents of type $l, \varphi_{l}(\sigma)=w_{e} \geq \nu(\sigma)$, or

$$
w_{l}-t_{l}=w_{e}=\left(\chi_{l}^{e} \mu_{l}\right)^{-1}\left(\chi_{l}^{p} \mu_{l} t_{l}+\mu_{h} t_{l}\right) \geq\left(1-\chi_{l}^{e} \mu_{l}\right)\left[\chi_{l}^{p} \mu_{l}\left(w_{l}-t_{l}\right)+\mu_{h}\left(w_{h}-t_{l}-\phi\right)\right] .
$$

Let $\epsilon=\frac{\mu_{h}}{\mu_{l}+\mu_{h}} \phi$ and let $\hat{\sigma}$ be given by $\hat{\chi}_{i}^{j}=\chi_{i}^{j}$ for all $i, j, \hat{t}_{h}=t_{l}+\phi-\epsilon, \hat{t}_{l}=t_{l}-\epsilon$, and $\hat{w}_{e}=w_{e}+\epsilon$. Then, producers of type $h$ do not hide income as $w_{h}-\hat{t}_{h}=w_{h}-t_{l}-\phi+\epsilon=$ $w_{h}-\hat{t}_{l}-\phi$, which is also greater than $w_{l}-\hat{t}_{l}$, because $w_{h}-w_{l}>\phi$, so that $\varphi_{h}(\hat{\sigma})>\varphi_{l}(\hat{\sigma})$. Also, $\varphi_{l}(\hat{\sigma})=w_{l}-\hat{t}_{l}=w_{l}-t_{l}+\epsilon=w_{e}+\epsilon=\hat{w}_{e}$ and the tax receipts increase to

$\chi_{l}^{p} \mu_{l} \hat{t}_{l}+\mu_{h} \hat{t}_{h}=\chi_{l}^{p} \mu_{l}\left(t_{l}-\epsilon\right)+\mu_{h}\left(t_{l}+\phi-\epsilon\right)=\chi_{l}^{p} \mu_{l} t_{l}+\mu_{h} t_{l}+\mu_{h} \phi-q \epsilon=\chi_{l}^{e} \mu_{l} w_{e}+(1-q) \epsilon$

or $\chi_{l}^{e} \mu_{l} w_{e}+\chi_{l}^{e} \mu_{l} \epsilon$, because $\mu_{h} \phi=\left(\mu_{l}+\mu_{h}\right) \epsilon=\epsilon$ from $\epsilon=\frac{\mu_{h}}{\mu_{l}+\mu_{h}} \phi$ and $1-q=1-\theta$, which is strictly greater than zero and exactly enough to pay all $\chi_{l}^{e} \mu_{l}$ enforcers $\hat{w}_{e}$, while

$$
\begin{aligned}
\nu(\hat{\sigma}) & =\left(1-\chi_{l}^{e} \mu_{l}\right)\left[\chi_{l}^{p} \mu_{l}\left(w_{l}-t_{l}+\epsilon\right)+\mu_{h}\left(w_{h}-t_{l}-\phi+\epsilon\right)\right] \\
& =\left(1-\chi_{l}^{e} \mu_{l}\right)\left[\chi_{l}^{p} \mu_{l}\left(w_{l}-t_{l}\right)+\mu_{h}\left(w_{h}-t_{l}-\phi\right)\right]+\left(1-\chi_{l}^{e} \mu_{l}\right) q \epsilon<\nu(\sigma)+\epsilon .
\end{aligned}
$$

That is, $\varphi_{l}(\hat{\sigma})=\hat{w}_{e}>\nu(\hat{\sigma})$ so that $\hat{\sigma}$ satisfies all constraints in Problem (PP) but, saving the cost of hiding income, yields a higher objective function value, $\chi_{l}^{p} \mu_{l} w_{l}+\mu_{h} w_{h}$, than $\sigma$, and thus dominates it.

Second, suppose $\chi_{l}^{a}>0$. Then, $p>0$ and, as $\sigma$ obeys all constraints, $\varphi_{l}(\sigma)=w_{e}=\nu(\sigma)$ or

$$
(1-\theta p)\left(w_{l}-t_{l}\right)=w_{e}=\left(\chi_{l}^{e} \mu_{l}\right)^{-1}\left(\chi_{l}^{p} \mu_{l} t_{l}+\mu_{h} t_{l}\right)=\theta\left[\chi_{l}^{p} \mu_{l}\left(w_{l}-t_{l}\right)+\mu_{h}\left(w_{h}-t_{l}-\phi\right)\right] .
$$

Let $\epsilon_{l}=\frac{\mu_{h}}{\mu_{l}+\mu_{h}} \phi(1+(1-\theta) \theta)^{-1}\left(\theta^{-1}-p\right)^{-1}>0$ and $\epsilon_{h}=\frac{\mu_{l}+\mu_{h}}{\mu_{h}}\left(\theta^{-1}-p-\chi_{l}^{p} \mu_{l}\right) \epsilon_{l}$ and let $\hat{\sigma}$ be given by $\hat{\chi}_{i}^{j}=\chi_{i}^{j}$ for all $i, j, \hat{t}_{h}=t_{l}+\phi-\epsilon_{h}, \hat{t}_{l}=t_{l}-\epsilon_{l}$, and $\hat{w}_{e}=w_{e}+(1-\theta p) \epsilon_{l}$. Then, $\epsilon_{h}>\epsilon_{l}$ because $\mu_{h}^{-1}\left(\theta^{-1}-p-\chi_{l}^{p} \mu_{l}\right)>1$ as $\theta^{-1}>1>\chi_{l}^{a} \mu_{l}+\chi_{l}^{p} \mu_{l}+\mu_{h}$, so that producers of type $h$ do not hide income as $w_{h}-\hat{t}_{h}=w_{h}-t_{l}-\phi+\epsilon_{h}>w_{h}-t_{l}-\phi+\epsilon_{l}=w_{h}-\hat{t}_{l}-\phi$, which is also greater than $w_{l}-\hat{t}_{l}$, because $w_{h}-w_{l}>\phi$, so that $\varphi_{h}(\hat{\sigma})>\varphi_{l}(\hat{\sigma})$. Also, $\varphi_{l}(\hat{\sigma})=$ $(1-\theta p)\left(w_{l}-\hat{t}_{l}\right)=(1-\theta p)\left(w_{l}-t_{l}\right)+(1-\theta p) \epsilon_{l}=w_{e}+(1-\theta p) \epsilon_{l}=\hat{w}_{e}$ and the tax receipts increase to, using the definition of $\epsilon_{l}$ to replace $\mu_{h} \phi=\left(\mu_{l}+\mu_{h}\right)(1+(1-\theta) \theta)\left(\theta^{-1}-p\right) \epsilon_{l}$, the expression for $\epsilon_{h}$, the budget equation, and the fact that $(1-\theta)=\chi_{l}^{e} \mu_{l}$,

$$
\begin{aligned}
\chi_{l}^{p} \mu_{l} \hat{t}_{l}+\mu_{h} \hat{t}_{h} & =\chi_{l}^{p} \mu_{l}\left(t_{l}-\epsilon_{l}\right)+\mu_{h}\left(t_{l}+\phi-\epsilon_{h}\right) \\
& =\chi_{l}^{p} \mu_{l} t_{l}+\mu_{h} t_{l}+\mu_{h} \phi-\mu_{h} \epsilon_{h}-\chi_{l}^{p} \mu_{l} \epsilon_{l} \\
& =\left(\chi_{l}^{p} \mu_{l} t_{l}+\mu_{h} t_{l}\right)+(1+(1-\theta) \theta)\left(\theta^{-1}-p\right) \epsilon_{l}-\left(\theta^{-1}-p-\chi_{l}^{p} \mu_{l}\right) \epsilon_{l}-\chi_{l}^{p} \mu_{l} \epsilon_{l} \\
& =\chi_{l}^{e} \mu_{l} w_{e}+(1-\theta) \epsilon_{l}-(1-\theta) \theta p \epsilon_{l}+\chi_{l}^{p} \mu_{l} \epsilon_{l}-\chi_{l}^{p} \mu_{l} \epsilon_{l} \\
& =\chi_{l}^{e} \mu_{l} w_{e}+\chi_{l}^{e} \mu_{l}(1-\theta p) \epsilon_{l}
\end{aligned}
$$




$$
\begin{aligned}
& =\chi_{l}^{e} \mu_{l}\left(w_{e}+(1-\theta p) \epsilon_{l}\right) \\
& =\chi_{l}^{e} \mu_{l} \hat{w}_{e}
\end{aligned}
$$

which is strictly greater than zero and exactly enough to pay all $\chi_{l}^{e} \mu_{l}$ enforcers $\hat{w}_{e}$, while

$$
\begin{aligned}
\nu(\hat{\sigma}) & =\theta\left[\chi_{l}^{p} \mu_{l}\left(w_{l}-t_{l}+\epsilon_{l}\right)+\mu_{h}\left(w_{h}-t_{l}-\phi+\epsilon_{h}\right)\right] \\
& =\theta\left[\chi_{l}^{p} \mu_{l}\left(w_{l}-t_{l}\right)+\mu_{h}\left(w_{h}-t_{l}-\phi\right)\right]+\theta\left(\chi_{l}^{p} \mu_{l} \epsilon_{l}+\mu_{h} \epsilon_{h}\right) \\
& =\nu(\sigma)+\theta\left(\chi_{l}^{p} \mu_{l} \epsilon_{l}+\left(\theta^{-1}-p-\chi_{l}^{p} \mu_{l}\right) \epsilon_{l}\right) \\
& =\nu(\sigma)+(1-\theta p) \epsilon_{l} .
\end{aligned}
$$

That is, $\varphi_{l}(\hat{\sigma})=\hat{w}_{e}=\nu(\hat{\sigma})$ so that $\hat{\sigma}$ satisfies all constraints in Problem (PP) but, saving the cost of hiding income, yields a higher objective function value, $\chi_{l}^{p} \mu_{l} w_{l}+\mu_{h} w_{h}$, than $\sigma$, and thus dominates it, which completes the proof.

\section{Lemma 3}

Proof. Consider any regime $\sigma$ with $\chi_{h}^{p}=1, \chi_{l}^{p}>0$, and, by Lemma 2, $\chi_{l}^{e}>0$ that satisfies all constraints of Problem (PP). Following Proposition 4, $\sigma$ also induces $\zeta=w_{h}, \psi\left(w_{h}-\zeta\right)=0$, and $t(\zeta)=t_{h}$. First, as $\chi_{l}^{p}>0$ and $\chi_{l}^{e}>0$, combining constraints (8) and (9) gives

$$
\varphi_{l}(\sigma) \geq \max \left\{w_{e}, \nu(\sigma)\right\} \geq w_{e} \geq \max \left\{\varphi_{l}(\sigma), \nu(\sigma)\right\} \geq \varphi_{l}(\sigma)
$$

implying that $\varphi_{l}(\sigma)=w_{e}$. Second, if $\chi_{l}^{a}>0$, then the inequality in constraint (10) has to be satisfied and combining it with constraint (9) yields

$$
\nu(\sigma) \geq \max \left\{\varphi_{l}(\sigma), w_{e}\right\}=w_{e} \geq \max \left\{\varphi_{l}(\sigma), \nu(\sigma)\right\} \geq \nu(\sigma)
$$

implying that $\varphi_{l}(\sigma)=w_{e}=\nu(\sigma)$. Finally, suppose that $\chi_{l}^{a}=0$. Then, the inequality in constraint (10) may or may not be satisfied. Suppose that $w_{e}>\nu(\sigma)$. I show that there is another regime $\hat{\sigma}$ with $\hat{w}_{e}=\nu(\hat{\sigma})$ that is associated with higher welfare and thus dominates $\sigma$. Given the regime $\sigma, \chi_{l}^{e}=1-\chi_{l}^{p}>0, p=0, \theta=1-\chi_{l}^{e} \mu_{l}$, and $\varphi_{l}(\sigma)=w_{e}>\nu(\sigma)$ or

$$
w_{l}-t_{l}=w_{e}=\left(\chi_{l}^{e} \mu_{l}\right)^{-1}\left(\chi_{l}^{p} \mu_{l} t_{l}+\mu_{h} t_{h}\right)>\left(1-\chi_{l}^{e} \mu_{l}\right)\left[\chi_{l}^{p} \mu_{l}\left(w_{l}-t_{l}\right)+\mu_{h}\left(w_{h}-t_{h}\right)\right]>0 .
$$

The tax receipts implied by $\sigma$ are bounded away from zero by $\nu(\sigma)$ as, by inequality (20), $w_{h}-t_{h}>w_{l}-t_{l} \geq 0$. There exists an $\epsilon>0$ such that, using $q=\left(\chi_{l}^{p} \mu_{l}+\mu_{h}\right)$,

$$
w_{l}-t_{l}+\epsilon>\left(\chi_{l}^{e} \mu_{l}\right)^{-1}\left(\chi_{l}^{p} \mu_{l} t_{l}+\mu_{h} t_{h}-q \epsilon\right)>\left(1-\chi_{l}^{e} \mu_{l}\right)\left[\chi_{l}^{p} \mu_{l}\left(w_{l}-t_{l}\right)+\mu_{h}\left(w_{h}-t_{h}\right)+q \epsilon\right] .
$$


(The tax receipts are still bounded away from zero.) There exists a $\delta>0, \delta<\chi_{l}^{e}$ such that

$$
\begin{aligned}
w_{l}-t_{l}+\epsilon & >\left(\chi_{l}^{e} \mu_{l}-\delta \mu_{l}\right)^{-1}\left(\chi_{l}^{p} \mu_{l} t_{l}+\mu_{h} t_{h}-q \epsilon+\delta \mu_{l}\left(t_{l}-\epsilon\right)\right) \\
& >\left(1-\chi_{l}^{e} \mu_{l}+\delta \mu_{l}\right)\left[\chi_{l}^{p} \mu_{l}\left(w_{l}-t_{l}\right)+\mu_{h}\left(w_{h}-t_{h}\right)+q \epsilon+\delta \mu_{l}\left(w_{l}-t_{l}+\epsilon\right)\right] .
\end{aligned}
$$

(The tax receipts are still bounded away from zero.) Then, there exists a $\kappa>0$ such that

$$
\begin{aligned}
& w_{l}-t_{l}+\epsilon>\left(\chi_{l}^{e} \mu_{l}-\delta \mu_{l}\right)^{-1}\left(\chi_{l}^{p} \mu_{l} t_{l}+\mu_{h} t_{h}-q \epsilon+\delta \mu_{l}\left(t_{l}-\epsilon\right)-\mu_{h} \kappa\right) \\
& =\left(1-\chi_{l}^{e} \mu_{l}+\delta \mu_{l}\right)\left[\chi_{l}^{p} \mu_{l}\left(w_{l}-t_{l}\right)+\mu_{h}\left(w_{h}-t_{h}\right)+q \epsilon+\delta \mu_{l}\left(w_{l}-t_{l}+\epsilon\right)+\mu_{h} \kappa\right] .
\end{aligned}
$$

(The receipts are still positive.) Then, there are $\gamma_{l}>0$ and $\gamma_{h}=-\frac{\left(\chi_{l}^{p}+\delta\right) \mu_{l}}{\mu_{h}} \gamma_{l}<0$ such that

$$
\begin{aligned}
& w_{l}-t_{l}+\epsilon-\gamma_{l} \\
= & \left(\chi_{l}^{e} \mu_{l}-\delta \mu_{l}\right)^{-1}\left(\chi_{l}^{p} \mu_{l} t_{l}+\mu_{h} t_{h}-q \epsilon+\delta \mu_{l}\left(t_{l}-\epsilon\right)-\mu_{h} \kappa+\left(\chi_{l}^{p}+\delta\right) \mu_{l} \gamma_{l}+\mu_{h} \gamma_{h}\right) \\
= & \left(1-\chi_{l}^{e} \mu_{l}+\delta \mu_{l}\right)\left[\chi_{l}^{p} \mu_{l}\left(w_{l}-t_{l}\right)+\mu_{h}\left(w_{h}-t_{h}\right)+q \epsilon+\delta \mu_{l}\left(w_{l}-t_{l}+\epsilon\right)+\mu_{h} \kappa\right. \\
& \left.-\left(\chi_{l}^{p}+\delta\right) \mu_{l} \gamma_{l}-\mu_{h} \gamma_{h}\right],
\end{aligned}
$$

because $\left(\chi_{l}^{p}+\delta\right) \mu_{l} \gamma_{l}+\mu_{h} \gamma_{h}=0$, (The tax receipts have not changed.) or, more compactly,

$$
\begin{aligned}
& w_{l}-\left(t_{l}-\epsilon+\gamma_{l}\right) \\
= & \left(\left(\chi_{l}^{e}-\delta\right) \mu_{l}\right)^{-1}\left(\left(\chi_{l}^{p}+\delta\right) \mu_{l}\left(t_{l}-\epsilon+\gamma_{l}\right)+\mu_{h}\left(t_{h}-\epsilon-\kappa+\gamma_{h}\right)\right) \\
= & \left(1-\left(\chi_{l}^{e}-\delta\right) \mu_{l}\right)\left[\left(\chi_{l}^{p}+\delta\right) \mu_{l}\left(w_{l}-\left(t_{l}-\epsilon+\gamma_{l}\right)\right)+\mu_{h}\left(w_{h}-\left(t_{h}-\epsilon-\kappa+\gamma_{h}\right)\right)\right],
\end{aligned}
$$

or, even more compactly,

$$
w_{l}-\hat{t}_{l}=\left(\hat{\chi}_{l}^{e} \mu_{l}\right)^{-1}\left(\hat{\chi}_{l}^{p} \mu_{l} \hat{t}_{l}+\mu_{h} \hat{t}_{h}\right)=\left(1-\hat{\chi}_{l}^{e} \mu_{l}\right)\left[\hat{\chi}_{l}^{p} \mu_{l}\left(w_{l}-\hat{t}_{l}\right)+\mu_{h}\left(w_{h}-\hat{t}_{h}\right)\right]
$$

where $\hat{\chi}_{l}^{p}=\chi_{l}^{p}+\delta>0, \hat{\chi}_{l}^{e}=\chi_{l}^{e}-\delta>0, \hat{\chi}_{l}^{a}=\chi_{l}^{a}=0, \hat{t}_{l}=t_{l}-\epsilon+\gamma_{l}, \hat{t}_{h}=t_{h}-\epsilon-\kappa+\gamma_{h}$. Letting $\hat{w}_{e}=\left(\hat{\chi}_{l}^{e} \mu_{l}\right)^{-1}\left(\hat{\chi}_{l}^{p} \mu_{l} \hat{t}_{l}+\mu_{h} \hat{t}_{h}\right)$ and $\hat{\sigma}=\left(\hat{\chi}_{l}^{p}, \hat{\chi}_{l}^{e}, \hat{\chi}_{l}^{a}, 1,0,0, \hat{t}_{l}, \hat{t}_{h}, \hat{w}_{e}\right)$ gives $\varphi_{l}(\hat{\sigma})=$ $\hat{w}_{e}=\nu(\hat{\sigma})$. As $w_{h}-t_{h} \geq w_{h}-t_{l}-\phi$ by inequality (18), $w_{h}-\hat{t}_{h} \geq w_{h}-\hat{t}_{l}-\phi>w_{l}-\hat{t}_{l}$ is satisfied, implying both no hidden income and $\varphi_{h}(\hat{\sigma})>\varphi_{l}(\hat{\sigma})$, and the tax receipts are strictly positive. Therefore, $\hat{\sigma}$ satisfies all constraints of Problem (PP) but yields a higher objective function value than $\sigma$, because $\hat{\chi}_{l}^{p}=\chi_{l}^{p}+\delta>\chi_{l}^{p}$, which completes the proof.

\section{Lemma 4}

Proof. Consider any solution $\sigma$ to Problem $\left(\mathrm{PP}^{\prime}\right)$. From constraint (22) it has to hold that $t_{l}+\phi \geq t_{h}$. The following argument is independent of whether $p=0$ or $p>0$. Suppose for a contradiction that $t_{l}+\phi>t_{h}$, implying that $(1-\theta p)\left(w_{h}-t_{h}\right)>(1-\theta p)\left(w_{h}-t_{l}-\phi\right)$. Then, 
there exist an $\epsilon_{h}>0$ and (as $\chi_{l}^{p}>0$ ) an $\epsilon_{l}=-\frac{\mu_{h}}{\chi_{l}^{p} \mu_{l}} \epsilon_{h}<0$ such that $t_{l}+\phi+\epsilon_{l}=t_{h}+\epsilon_{h}$, or

$$
t_{l}+\phi=t_{h}+\left(1+\frac{\mu_{h}}{\chi_{l}^{p} \mu_{l}}\right) \epsilon_{h} .
$$

Let $\tilde{t}_{i}=t_{i}+\epsilon_{i}$. As $\tilde{t}_{l}+\phi=t_{l}+\phi+\epsilon_{l}=t_{h}+\epsilon_{h}=\tilde{t}_{h}$, as $\phi<\left(w_{h}-w_{l}\right)$,

$$
(1-\theta p)\left(w_{h}-\tilde{t}_{h}\right)=(1-\theta p)\left(w_{h}-\tilde{t}_{l}-\phi\right)>(1-\theta p)\left(w_{l}-\tilde{t}_{l}\right)
$$

and, starting from $\varphi_{l}(\sigma)=w_{e}=\nu(\sigma)$, as $\tilde{t}_{l}<t_{l}$, using $\epsilon_{l}=-\frac{\mu_{h}}{\chi_{l}^{p} \mu_{l}} \epsilon_{h}$ and $\theta=1-\chi_{l}^{e} \mu_{l}$,

$$
\begin{aligned}
(1-\theta p)\left(w_{l}-\tilde{t}_{l}\right)>w_{e} & =\left(\chi_{l}^{e} \mu_{l}\right)^{-1}\left(\chi_{l}^{p} \mu_{l} \tilde{t}_{l}+\mu_{h} \tilde{t}_{h}\right) \\
& =\left(\chi_{l}^{e} \mu_{l}\right)^{-1}\left(\chi_{l}^{p} \mu_{l} t_{l}+\mu_{h} t_{h}+\chi_{l}^{p} \mu_{l} \epsilon_{l}+\mu_{h} \epsilon_{h}\right) \\
& =\left(\chi_{l}^{e} \mu_{l}\right)^{-1}\left(\chi_{l}^{p} \mu_{l} t_{l}+\mu_{h} t_{h}\right) \\
& =\nu(\sigma) \\
& \left.=\left(1-\chi_{l}^{e} \mu_{l}\right)\left[\chi_{l}^{p} \mu_{l}\left(w_{l}-t_{l}\right)+\mu_{h}\left(w_{h}-t_{h}\right)\right)\right] \\
& =\left(1-\chi_{l}^{e} \mu_{l}\right)\left[\chi_{l}^{p} \mu_{l}\left(w_{l}-t_{l}\right)+\mu_{h}\left(w_{h}-t_{h}\right)-\chi_{l}^{p} \mu_{l} \epsilon_{l}-\mu_{h} \epsilon_{h}\right] \\
& =\left(1-\chi_{l}^{e} \mu_{l}\right)\left[\chi_{l}^{p} \mu_{l}\left(w_{l}-\tilde{t}_{l}\right)+\mu_{h}\left(w_{h}-\tilde{t}_{h}\right)\right],
\end{aligned}
$$

because $\chi_{l}^{p} \mu_{l} \epsilon_{l}+\mu_{h} \epsilon_{h}=0$. The tax receipts implied by $\sigma$ are bounded away from zero by $\nu(\sigma)$ as, by inequality (20), $w_{h}-t_{h}>w_{l}-t_{l} \geq 0$. In fact, due to $\varphi_{l}(\sigma)=w_{e}=\nu(\sigma)>0$, $w_{l}-t_{l}>0$. The last set of inequalities and equalities can be summarized as

$$
(1-\theta p)\left(w_{l}-\tilde{t}_{l}\right)>\left(\chi_{l}^{e} \mu_{l}\right)^{-1}\left(\chi_{l}^{p} \mu_{l} \tilde{t}_{l}+\mu_{h} \tilde{t}_{h}\right)=\left(1-\chi_{l}^{e} \mu_{l}\right)\left[\chi_{l}^{p} \mu_{l}\left(w_{l}-\tilde{t}_{l}\right)+\mu_{h}\left(w_{h}-\tilde{t}_{h}\right)\right]>0 .
$$

Then, there exists a $\gamma>0, \gamma<w_{l}-\tilde{t}_{l}\left(<w_{h}-\tilde{t}_{h}\right.$ by $\left.(27)\right)$, such that, letting $\bar{t}_{i}=\tilde{t}_{i}+\gamma$,

$$
(1-\theta p)\left(w_{l}-\bar{t}_{l}\right)>\left(\chi_{l}^{e} \mu_{l}\right)^{-1}\left(\chi_{l}^{p} \mu_{l} \bar{t}_{l}+\mu_{h} \bar{t}_{h}\right)>\left(1-\chi_{l}^{e} \mu_{l}\right)\left[\chi_{l}^{p} \mu_{l}\left(w_{l}-\bar{t}_{l}\right)+\mu_{h}\left(w_{h}-\bar{t}_{h}\right)\right]>0 .
$$

Then, irrespective of whether $p=0$ or $p>0$, there exists a $\bar{\delta}_{1}>0, \bar{\delta}_{1}<\chi_{l}^{e}$, such that, using $\theta=1-\chi_{l}^{e} \mu_{l}$, for all $\delta_{1} \in\left[0, \bar{\delta}_{1}\right]$,

$$
\left(1-\left(1-\left(\chi_{l}^{e}-\delta_{1}\right) \mu_{l}\right) p\right)\left(w_{l}-\bar{t}_{l}\right)>\left(\left(\chi_{l}^{e}-\delta_{1}\right) \mu_{l}\right)^{-1}\left(\left(\chi_{l}^{p}+\delta_{1}\right) \mu_{l} \bar{t}_{l}+\mu_{h} \bar{t}_{h}\right),
$$

(note: $\bar{t}_{l}$ could be negative) as well as a $\bar{\delta}_{2}>0, \bar{\delta}_{2}<\chi_{l}^{e}$, such that, for all $\delta_{2} \in\left[0, \bar{\delta}_{2}\right]$,

$$
\begin{aligned}
& \left(\left(\chi_{l}^{e}-\delta_{2}\right) \mu_{l}\right)^{-1}\left(\left(\chi_{l}^{p}+\delta_{2}\right) \mu_{l} \bar{t}_{l}+\mu_{h} \bar{t}_{h}\right) \\
& >\left(1-\left(\chi_{l}^{e}-\delta_{2}\right) \mu_{l}\right)\left[\left(\chi_{l}^{p}+\delta_{2}\right) \mu_{l}\left(w_{l}-\bar{t}_{l}\right)+\mu_{h}\left(w_{h}-\bar{t}_{h}\right)\right]>0 .
\end{aligned}
$$


Let $\delta=\min \left\{\bar{\delta}_{1}, \bar{\delta}_{2}\right\}$. Then,

$$
\begin{aligned}
\left(1-\left(1-\left(\chi_{l}^{e}-\delta\right) \mu_{l}\right) p\right)\left(w_{l}-\bar{t}_{l}\right) & >\left(\left(\chi_{l}^{e}-\delta\right) \mu_{l}\right)^{-1}\left(\left(\chi_{l}^{p}+\delta\right) \mu_{l} \bar{t}_{l}+\mu_{h} \bar{t}_{h}\right) \\
& >\left(1-\left(\chi_{l}^{e}-\delta\right) \mu_{l}\right)\left[\left(\chi_{l}^{p}+\delta\right) \mu_{l}\left(w_{l}-\bar{t}_{l}\right)+\mu_{h}\left(w_{h}-\bar{t}_{h}\right)\right]>0
\end{aligned}
$$

Let $\hat{\chi}_{l}^{p}=\chi_{l}^{p}+\delta>0, \hat{\chi}_{l}^{e}=\chi_{l}^{e}-\delta>0, \hat{\chi}_{l}^{a}=\chi_{l}^{a} \geq 0, \hat{p}=\hat{\chi}_{l}^{a} \mu_{l}=\chi_{l}^{a} \mu_{l}=p$, and $\hat{\theta}=\left(1-\hat{\chi}_{l}^{e} \mu_{l}\right)$. Then, there exists an $\eta>0$ such that, letting $\breve{t}_{i}=\bar{t}_{i}-\eta$,

$$
(1-\hat{\theta} \hat{p})\left(w_{l}-\breve{t}_{l}\right)>\left(\hat{\chi}_{l}^{e} \mu_{l}\right)^{-1}\left(\hat{\chi}_{l}^{p} \mu_{l} \breve{t}_{l}+\mu_{h} \breve{t}_{h}\right)=\hat{\theta}\left[\hat{\chi}_{l}^{p} \mu_{l}\left(w_{l}-\breve{t}_{l}\right)+\mu_{h}\left(w_{h}-\breve{t}_{h}\right)\right]>0
$$

Then, there are a $\kappa_{l}>0$ and a $\kappa_{h}=-\frac{\hat{\chi}_{l}^{p} \mu_{l}}{\mu_{h}} \kappa_{l}<0$ such that, letting $\hat{t}_{i}=\breve{t}_{i}+\kappa_{i}$,

$$
\begin{aligned}
(1-\hat{\theta} \hat{p})\left(w_{l}-\hat{t}_{l}\right) & =\left(\hat{\chi}_{l}^{e} \mu_{l}\right)^{-1}\left(\hat{\chi}_{l}^{p} \mu_{l} \hat{t}_{l}+\mu_{h} \hat{t}_{h}\right) \\
& =\left(\hat{\chi}_{l}^{e} \mu_{l}\right)^{-1}\left(\hat{\chi}_{l}^{p} \mu_{l} \breve{l}_{l}+\mu_{h} \breve{t}_{h}+\hat{\chi}_{l}^{p} \mu_{l} \kappa_{l}+\mu_{h} \kappa_{h}\right) \\
& =\left(\hat{\chi}_{l}^{e} \mu_{l}\right)^{-1}\left(\hat{\chi}_{l}^{p} \mu_{l} \breve{l}_{l}+\mu_{h} \breve{t}_{h}\right) \\
& =\hat{\theta}\left[\hat{\chi}_{l}^{p} \mu_{l}\left(w_{l}-\breve{t}_{l}\right)+\mu_{h}\left(w_{h}-\breve{t}_{h}\right)\right] \\
& =\hat{\theta}\left[\hat{\chi}_{l}^{p} \mu_{l}\left(w_{l}-\breve{t}_{l}\right)+\mu_{h}\left(w_{h}-\breve{t}_{h}\right)-\hat{\chi}_{l}^{p} \mu_{l} \kappa_{l}-\mu_{h} \kappa_{h}\right] \\
& =\hat{\theta}\left[\hat{\chi}_{l}^{p} \mu_{l}\left(w_{l}-\hat{t}_{l}\right)+\mu_{h}\left(w_{h}-\hat{t}_{h}\right)\right]>0,
\end{aligned}
$$

because $\hat{\chi}_{l}^{p} \mu_{l} \kappa_{l}+\mu_{h} \kappa_{h}=0$, or, summarizing these equations,

$$
(1-\hat{\theta} \hat{p})\left(w_{l}-\hat{t}_{l}\right)=\left(\hat{\chi}_{l}^{e} \mu_{l}\right)^{-1}\left(\hat{\chi}_{l}^{p} \mu_{l} \hat{t}_{l}+\mu_{h} \hat{t}_{h}\right)=\hat{\theta}\left[\hat{\chi}_{l}^{p} \mu_{l}\left(w_{l}-\hat{t}_{l}\right)+\mu_{h}\left(w_{h}-\hat{t}_{h}\right)\right]>0
$$

Letting $\hat{w}_{e}=\left(\hat{\chi}_{l}^{e} \mu_{l}\right)^{-1}\left(\hat{\chi}_{l}^{p} \mu_{l} \hat{t}_{l}+\mu_{h} \hat{t}_{h}\right)$ and $\hat{\sigma}=\left(\hat{\chi}_{l}^{p}, \hat{\chi}_{l}^{e}, \hat{\chi}_{l}^{a}, 1,0,0, \hat{t}_{l}, \hat{t}_{h}, \hat{w}_{e}\right)$, the last equation implies $\varphi_{l}(\hat{\sigma})=\hat{w}_{e}=\nu(\hat{\sigma})$. The tax receipts are still bounded away from zero. Since $t_{l}+\epsilon_{l}+\phi=t_{h}+\epsilon_{h}$, as established in the very beginning, and $\kappa_{l}>0$ while $\kappa_{h}<0$, it holds that $t_{l}+\epsilon_{l}+\gamma-\eta+\kappa_{l}+\phi>t_{h}+\epsilon_{h}+\gamma-\eta+\kappa_{h}$ so that $\hat{t}_{l}+\phi>\hat{t}_{h}$. Hence, constraint (22) is satisfied and $w_{h}-\hat{t}_{h}>w_{l}-\hat{t}_{l}$. Therefore, $\hat{\sigma}$ satisfies all constraints of Problem $\left(\mathrm{PP}^{\prime}\right)$ but yields a higher objective function value than $\sigma$, because $\hat{\chi}_{l}^{p}=\chi_{l}^{p}+\delta>\chi_{l}^{p}$, a contradiction.

\section{Proposition 5}

Proof. Consider any solution $\sigma$ to Problem $\left(\mathrm{PP}^{\prime}\right)$ and suppose for a contradiction that some agents of type $l$ engage in appropriation. I show in two steps that there is another regime in the constraint set that attains a higher objective function value and does not have any appropriators. First, starting from $\sigma$, I show that the planner can reallocate all appropriators to enforcement and find a tax increase for all producers as well as a wage in enforcement such that no agent wants to deviate to an occupation different from the amended occupation prescriptions. Second, starting from this amended regime, I show that the planner can reallocate 
some of these enforcers to production and find a tax schedule as well as a wage in enforcement such that all constraints of Problem $\left(\mathrm{PP}^{\prime}\right)$ are satisfied, while more agents produce, which increases the objective function value.

As $\chi_{l}^{p}>0, \chi_{l}^{e}>0$ by Lemma 2. Following Lemma $4, t_{h}=t_{l}+\phi$. Then, writing out the payoffs $\varphi_{l}(\sigma)$ and $\nu(\sigma)$ in constraints (23) and (24), using constraint (26) to replace $p$ with $\chi_{l}^{a} \mu_{l}$ and $\chi_{l}^{e} \mu_{l}$ with $(1-\theta)$ and constraint $(25)$ to replace $\chi_{l}^{a}$ with $1-\chi_{l}^{p}-\chi_{l}^{e}$, so that $p=\chi_{l}^{a} \mu_{l}=$ $\left(1-\chi_{l}^{p}-\chi_{l}^{e}\right) \mu_{l}=\mu_{l}-\chi_{l}^{p} \mu_{l}-(1-\theta)=\theta+\mu_{l}-\chi_{l}^{p} \mu_{l}-\mu_{l}-\mu_{h}=\theta-\left(\chi_{l}^{p} \mu_{l}+\mu_{h}\right)=\theta-q$, where the last equality follows from constraint (26), the solution $\sigma$ to Problem $\left(\mathrm{PP}^{\prime}\right)$ satisfies

$$
\begin{aligned}
& t_{h}=t_{l}+\phi, \\
& (1-\theta(\theta-q))\left(w_{l}-t_{l}\right)=w_{e}, \\
& (1-\theta) w_{e}=\left(\chi_{l}^{p} \mu_{l} t_{l}+\mu_{h} t_{h}\right), \\
& w_{e}=\theta\left[\chi_{l}^{p} \mu_{l}\left(w_{l}-t_{l}\right)+\mu_{h}\left(w_{h}-t_{h}\right)\right], \\
& \theta-q \geq 0 .
\end{aligned}
$$

Inequality (32) ensures that $\chi_{l}^{a} \geq 0$. The assumption that some agents of type $l$ engage in appropriation implies that $\chi_{l}^{a}>0$ and thus $\theta>q$. Combining equations (29)-(31), we have

$$
(1-\theta(\theta-q))\left(w_{l}-t_{l}\right)=(1-\theta)^{-1}\left(\chi_{l}^{p} \mu_{l} t_{l}+\mu_{h} t_{h}\right)=\theta\left[\chi_{l}^{p} \mu_{l}\left(w_{l}-t_{l}\right)+\mu_{h}\left(w_{h}-t_{h}\right)\right]>0,
$$

where $w_{e}=(1-\theta)^{-1}\left(\chi_{l}^{p} \mu_{l} t_{l}+\mu_{h} t_{h}\right)$ from (30), as $\chi_{l}^{e}>0$ implies that $(1-\theta)>0$. All payoffs are strictly positive as $w_{h}-t_{h}>w_{l}-t_{l} \geq 0$ by equation (20), implying that $\theta\left[\chi_{l}^{p} \mu_{l}\left(w_{l}-t_{l}\right)+\mu_{h}\left(w_{h}-t_{h}\right)\right]>0$, and thus $w_{l}-t_{l}>0$.

Step 1. First, reallocating all $(\theta-q)$ appropriators to enforcement, keeping all other agents in their occupation, implies that $\tilde{\theta}=q<\theta, 1-\tilde{\theta}=1-q>1-\theta$, so that the payoffs satisfy

$$
w_{l}-t_{l}>\max \left\{(1-q)^{-1}\left(\chi_{l}^{p} \mu_{l} t_{l}+\mu_{h} t_{h}\right), q\left[\chi_{l}^{p} \mu_{l}\left(w_{l}-t_{l}\right)+\mu_{h}\left(w_{h}-t_{h}\right)\right]\right\}>0
$$

because, compared to (33), $1>(1-\theta(\theta-q)),(1-q)^{-1}<(1-\theta)^{-1}$, and $q<\theta$. There are two cases: either, case (a), $\theta(1-\theta) \geq q(1-q)$ or, case (b), $\theta(1-\theta)<q(1-q)$.

Case (a). Suppose that $\theta(1-\theta) \geq q(1-q)$. In this case, equation (34) can be written as

$$
w_{l}-t_{l}>(1-q)^{-1}\left(\chi_{l}^{p} \mu_{l} t_{l}+\mu_{h} t_{h}\right) \geq q\left[\chi_{l}^{p} \mu_{l}\left(w_{l}-t_{l}\right)+\mu_{h}\left(w_{h}-t_{h}\right)\right],
$$

because from (33),

$$
\left(\chi_{l}^{p} \mu_{l} t_{l}+\mu_{h} t_{h}\right)=(1-\theta) \theta\left[\chi_{l}^{p} \mu_{l}\left(w_{l}-t_{l}\right)+\mu_{h}\left(w_{h}-t_{h}\right)\right]
$$


so that

$$
\begin{aligned}
(1-q)^{-1}\left(\chi_{l}^{p} \mu_{l} t_{l}+\mu_{h} t_{h}\right) & =(1-q)^{-1}(1-\theta) \theta\left[\chi_{l}^{p} \mu_{l}\left(w_{l}-t_{l}\right)+\mu_{h}\left(w_{h}-t_{h}\right)\right] \\
& \geq q\left[\chi_{l}^{p} \mu_{l}\left(w_{l}-t_{l}\right)+\mu_{h}\left(w_{h}-t_{h}\right)\right]
\end{aligned}
$$

as $\theta(1-\theta) \geq q(1-q)$. Therefore, from (35), there exists an $\epsilon>0$ such that

$$
w_{l}-t_{l}-\epsilon=(1-q)^{-1}\left(\chi_{l}^{p} \mu_{l}\left(t_{l}+\epsilon\right)+\mu_{h}\left(t_{h}+\epsilon\right)\right)>q\left[\chi_{l}^{p} \mu_{l}\left(w_{l}-t_{l}-\epsilon\right)+\mu_{h}\left(w_{h}-t_{h}-\epsilon\right)\right] .
$$

All payoffs are still strictly positive as $\left(\chi_{l}^{p} \mu_{l} t_{l}+\mu_{h} t_{h}\right)>0$ from (33), so that, for $\epsilon>0$, $\left(\chi_{l}^{p} \mu_{l}\left(t_{l}+\epsilon\right)+\mu_{h}\left(t_{h}+\epsilon\right)\right)>0$, implying that $w_{h}-t_{h}-\epsilon>w_{l}-t_{l}-\epsilon>0$. That is, taxing all producers that additional $\epsilon>0$ allows to reallocate all those who were assigned to appropriation in the original regime $\sigma$ to enforcement, and to pay all, now $(1-q)$, enforcement personnel the wages required, i.e., $(1-q)^{-1}\left(\chi_{l}^{p} \mu_{l}\left(t_{l}+\epsilon\right)+\mu_{h}\left(t_{h}+\epsilon\right)\right)$, such that no agent wants to deviate to appropriation and all agents of type $l$ are indifferent between production and enforcement.

Case (b). Suppose that $\theta(1-\theta)<q(1-q)$. In this case, equation (34) can be written as

$$
w_{l}-t_{l}>q\left[\chi_{l}^{p} \mu_{l}\left(w_{l}-t_{l}\right)+\mu_{h}\left(w_{h}-t_{h}\right)\right]>(1-q)^{-1}\left(\chi_{l}^{p} \mu_{l} t_{l}+\mu_{h} t_{h}\right),
$$

by a similar argument as above. There exists an $\epsilon_{1}>0$ such that, using $q=\chi_{l}^{p} \mu_{l}+\mu_{h}$,

$$
\begin{aligned}
w_{l}-t_{l}-\epsilon_{1} & =(1-q)^{-1}\left(\chi_{l}^{p} \mu_{l}\left(t_{l}+\epsilon_{1}\right)+\mu_{h}\left(t_{h}+\epsilon_{1}\right)\right) \\
& =(1-q)^{-1}\left(\chi_{l}^{p} \mu_{l} t_{l}+\mu_{h} t_{h}\right)+(1-q)^{-1} q \epsilon_{1} .
\end{aligned}
$$

Multiplying by $(1-q)$ and using $\left(\chi_{l}^{p} \mu_{l} t_{l}+\mu_{h} t_{h}\right)=(1-\theta)(1-\theta(\theta-q))\left(w_{l}-t_{l}\right)$ from (33),

$$
\epsilon_{1}=[(1-q)-(1-\theta)(1-\theta(\theta-q))]\left(w_{l}-t_{l}\right)
$$

Similarly, there exists an $\epsilon_{2}>0$ such that, again using $q=\chi_{l}^{p} \mu_{l}+\mu_{h}$,

$$
\begin{aligned}
(1-q)^{-1}\left(\chi_{l}^{p} \mu_{l} t_{l}+\mu_{h} t_{h}\right)+(1-q)^{-1} q \epsilon_{2} & =(1-q)^{-1}\left(\chi_{l}^{p} \mu_{l}\left(t_{l}+\epsilon_{2}\right)+\mu_{h}\left(t_{h}+\epsilon_{2}\right)\right) \\
& =q\left[\chi_{l}^{p} \mu_{l}\left(w_{l}-t_{l}-\epsilon_{2}\right)+\mu_{h}\left(w_{h}-t_{h}-\epsilon_{2}\right)\right] \\
& =q\left[\chi_{l}^{p} \mu_{l}\left(w_{l}-t_{l}\right)+\mu_{h}\left(w_{h}-t_{h}\right)\right]-q^{2} \epsilon_{2} .
\end{aligned}
$$

Multiplying both sides by $(1-q)$ and collecting terms gives

$$
\left(q+(1-q) q^{2}\right) \epsilon_{2}=(1-q) q\left[\chi_{l}^{p} \mu_{l}\left(w_{l}-t_{l}\right)+\mu_{h}\left(w_{h}-t_{h}\right)\right]-\left(\chi_{l}^{p} \mu_{l} t_{l}+\mu_{h} t_{h}\right),
$$


and using $\left(\chi_{l}^{p} \mu_{l} t_{l}+\mu_{h} t_{h}\right)=(1-\theta)(1-\theta(\theta-q))\left(w_{l}-t_{l}\right)$ and $\left[\chi_{l}^{p} \mu_{l}\left(w_{l}-t_{l}\right)+\mu_{h}\left(w_{h}-t_{h}\right)\right]=$ $\theta^{-1}(1-\theta(\theta-q))\left(w_{l}-t_{l}\right)$ from $(33)$ gives

$$
\epsilon_{2}=\frac{1}{q(1+(1-q) q)}\left[(1-q) q \theta^{-1}-(1-\theta)\right](1-\theta(\theta-q))\left(w_{l}-t_{l}\right) .
$$

We have that $\epsilon_{1}>\epsilon_{2}$, because

$$
[(1-q)-(1-\theta)(1-\theta(\theta-q))]>\frac{1}{q(1+(1-q) q)}\left[(1-q) q \theta^{-1}-(1-\theta)\right](1-\theta(\theta-q)) .
$$

To verify this claim, multiply both sides by $\theta q(1+(1-q) q)$ to get

$$
(1+(1-q) q)[\theta q(1-q)-q \theta(1-\theta)(1-\theta(\theta-q))]>[(1-q) q-\theta(1-\theta)](1-\theta(\theta-q))
$$

and note that, as $q \in(0,1)$ and $(1-\theta(\theta-q))<1$,

$$
\begin{aligned}
& (1+(1-q) q)[\theta q(1-q)-q \theta(1-\theta)(1-\theta(\theta-q))] \\
> & {[\theta q(1-q)-q \theta(1-\theta)(1-\theta(\theta-q))] } \\
> & {[\theta q(1-q)-q \theta(1-\theta)] } \\
> & {[(1-q) q-\theta(1-\theta)] } \\
> & {[(1-q) q-\theta(1-\theta)](1-\theta(\theta-q)), }
\end{aligned}
$$

where the third inequality holds because, as $q \in(0,1)$ and $1-\theta>0$,

$$
\begin{array}{r}
\theta q(1-q)-q \theta(1-\theta)>(1-q) q-\theta(1-\theta) \\
\Leftrightarrow \quad \theta(1-\theta)-q \theta(1-\theta)>(1-q) q-\theta q(1-q) \\
\Leftrightarrow \quad \theta(1-\theta)(1-q)>(1-q) q(1-\theta) \\
\Leftrightarrow \quad \theta>q,
\end{array}
$$

which holds by assumption. Therefore, as $\epsilon_{1}>\epsilon_{2}$,

$$
\begin{aligned}
w_{l}-t_{l}-\epsilon_{1} & =(1-q)^{-1}\left(\chi_{l}^{p} \mu_{l}\left(t_{l}+\epsilon_{1}\right)+\mu_{h}\left(t_{h}+\epsilon_{1}\right)\right) \\
& >(1-q)^{-1}\left(\chi_{l}^{p} \mu_{l}\left(t_{l}+\epsilon_{2}\right)+\mu_{h}\left(t_{h}+\epsilon_{2}\right)\right) \\
& =q\left[\chi_{l}^{p} \mu_{l}\left(w_{l}-t_{l}-\epsilon_{2}\right)+\mu_{h}\left(w_{h}-t_{h}-\epsilon_{2}\right)\right] \\
& >q\left[\chi_{l}^{p} \mu_{l}\left(w_{l}-t_{l}-\epsilon_{1}\right)+\mu_{h}\left(w_{h}-t_{h}-\epsilon_{1}\right)\right],
\end{aligned}
$$


so that there is an $\epsilon=\epsilon_{1}>0$ such that

$$
w_{l}-t_{l}-\epsilon=(1-q)^{-1}\left(\chi_{l}^{p} \mu_{l}\left(t_{l}+\epsilon\right)+\mu_{h}\left(t_{h}+\epsilon\right)\right)>q\left[\chi_{l}^{p} \mu_{l}\left(w_{l}-t_{l}-\epsilon\right)+\mu_{h}\left(w_{h}-t_{h}-\epsilon\right)\right] .
$$

Again, all payoffs are still strictly positive as $\left(\chi_{l}^{p} \mu_{l} t_{l}+\mu_{h} t_{h}\right)>0$ from (33), so that, for $\epsilon>0,\left(\chi_{l}^{p} \mu_{l}\left(t_{l}+\epsilon\right)+\mu_{h}\left(t_{h}+\epsilon\right)\right)>0$, implying that $w_{h}-t_{h}-\epsilon>w_{l}-t_{l}-\epsilon>0$. That is, again, taxing all producers that additional $\epsilon>0$ allows to reallocate all those who were assigned to appropriation in the original regime $\sigma$ to enforcement, and to pay all, now $(1-q)$, enforcement personnel the wages required, i.e., $(1-q)^{-1}\left(\chi_{l}^{p} \mu_{l}\left(t_{l}+\epsilon\right)+\mu_{h}\left(t_{h}+\epsilon\right)\right)$, such that no agent wants to deviate to appropriation and all agents of type $l$ are indifferent between production and enforcement. As summarized by equations (36) and (38), irrespective of whether $\theta(1-\theta) \geq q(1-q)$ or $\theta(1-\theta)<q(1-q)$, there exists an $\epsilon>0$ such that increasing the taxes for all producers by that amount allows to support the wage bill for all enforcers, once all appropriators are reallocated to enforcement, and no agent wants to deviate from the planner's amended occupational prescriptions.

Step 2. Second, starting from the planner's amended occupational prescriptions and taxes that satisfy (38), which is the same as (36), there is a tax decrease $\gamma>0$ such that

$$
\begin{aligned}
w_{l}-t_{l}-\epsilon+\gamma & >(1-q)^{-1}\left(\chi_{l}^{p} \mu_{l}\left(t_{l}+\epsilon-\gamma\right)+\mu_{h}\left(t_{h}+\epsilon-\gamma\right)\right) \\
& >q\left[\chi_{l}^{p} \mu_{l}\left(w_{l}-t_{l}-\epsilon+\gamma\right)+\mu_{h}\left(w_{h}-t_{h}-\epsilon+\gamma\right)\right]>0 .
\end{aligned}
$$

Then, using $q=\chi_{l}^{p} \mu_{l}+\mu_{h}$, there exists a $\bar{\delta}_{1}>0, \bar{\delta}_{1}<\chi_{l}^{e}$, such that, for all $\delta_{1} \in\left[0, \bar{\delta}_{1}\right]$,

$$
w_{l}-t_{l}-\epsilon+\gamma>\left(1-\left(\left(\chi_{l}^{p}+\delta_{1}\right) \mu_{l}+\mu_{h}\right)\right)^{-1}\left(\left(\chi_{l}^{p}+\delta_{1}\right) \mu_{l}\left(t_{l}+\epsilon-\gamma\right)+\mu_{h}\left(t_{h}+\epsilon-\gamma\right)\right)
$$

(note: $t_{l}+\epsilon-\gamma$ could be negative) as well as a $\bar{\delta}_{2}>0, \bar{\delta}_{2}<\chi_{l}^{e}$, such that, for all $\delta_{2} \in\left[0, \bar{\delta}_{2}\right]$,

$$
\begin{gathered}
\left(1-\left(\left(\chi_{l}^{p}+\delta_{2}\right) \mu_{l}+\mu_{h}\right)\right)^{-1}\left(\left(\chi_{l}^{p}+\delta_{2}\right) \mu_{l}\left(t_{l}+\epsilon-\gamma\right)+\mu_{h}\left(t_{h}+\epsilon-\gamma\right)\right) \\
>\left(\left(\chi_{l}^{p}+\delta_{2}\right) \mu_{l}+\mu_{h}\right)\left[\left(\chi_{l}^{p}+\delta_{2}\right) \mu_{l}\left(w_{l}-t_{l}-\epsilon+\gamma\right)+\mu_{h}\left(w_{h}-t_{h}-\epsilon+\gamma\right)\right] .
\end{gathered}
$$

Then, letting $\delta=\min \left\{\bar{\delta}_{1}, \bar{\delta}_{2}\right\}>0$, so that $0<\delta<\chi_{l}^{e}$, reallocating enforcers to production,

$$
\begin{aligned}
w_{l}-t_{l}-\epsilon+\gamma & >\left(1-\left(\left(\chi_{l}^{p}+\delta\right) \mu_{l}+\mu_{h}\right)\right)^{-1}\left(\left(\chi_{l}^{p}+\delta\right) \mu_{l}\left(t_{l}+\epsilon-\gamma\right)+\mu_{h}\left(t_{h}+\epsilon-\gamma\right)\right) \\
& >\left(\left(\chi_{l}^{p}+\delta\right) \mu_{l}+\mu_{h}\right)\left[\left(\chi_{l}^{p}+\delta\right) \mu_{l}\left(w_{l}-t_{l}-\epsilon+\gamma\right)+\mu_{h}\left(w_{h}-t_{h}-\epsilon+\gamma\right)\right] .
\end{aligned}
$$

Now, there is a tax decrease $\eta>0$ such that

$$
w_{l}-t_{l}-\epsilon+\gamma+\eta
$$




$$
\begin{aligned}
& >\left(1-\left(\left(\chi_{l}^{p}+\delta\right) \mu_{l}+\mu_{h}\right)\right)^{-1}\left(\left(\chi_{l}^{p}+\delta\right) \mu_{l}\left(t_{l}+\epsilon-\gamma-\eta\right)+\mu_{h}\left(t_{h}+\epsilon-\gamma-\eta\right)\right) \\
& =\left(\left(\chi_{l}^{p}+\delta\right) \mu_{l}+\mu_{h}\right)\left[\left(\chi_{l}^{p}+\delta\right) \mu_{l}\left(w_{l}-t_{l}-\epsilon+\gamma+\eta\right)+\mu_{h}\left(w_{h}-t_{h}-\epsilon+\gamma+\eta\right)\right]>0 .
\end{aligned}
$$

Then, there are a tax changes $\kappa_{l}>0$ and $\kappa_{h}=-\frac{\left(\chi_{l}^{p}+\delta\right) \mu_{l}}{\mu_{h}} \kappa_{l}<0$ such that

$$
\begin{aligned}
& w_{l}-t_{l}-\epsilon+\gamma+\eta-\kappa_{l} \\
= & \left(1-\left(\left(\chi_{l}^{p}+\delta\right) \mu_{l}+\mu_{h}\right)\right)^{-1}\left(\left(\chi_{l}^{p}+\delta\right) \mu_{l}\left(t_{l}+\epsilon-\gamma-\eta+\kappa_{l}\right)+\mu_{h}\left(t_{h}+\epsilon-\gamma-\eta+\kappa_{h}\right)\right) \\
= & \left(1-\left(\left(\chi_{l}^{p}+\delta\right) \mu_{l}+\mu_{h}\right)\right)^{-1}\left(\left(\chi_{l}^{p}+\delta\right) \mu_{l}\left(t_{l}+\epsilon-\gamma-\eta\right)+\mu_{h}\left(t_{h}+\epsilon-\gamma-\eta\right)\right. \\
& \left.+\left(\chi_{l}^{p}+\delta\right) \mu_{l} \kappa_{l}+\mu_{h} \kappa_{h}\right) \\
= & \left(1-\left(\left(\chi_{l}^{p}+\delta\right) \mu_{l}+\mu_{h}\right)\right)^{-1}\left(\left(\chi_{l}^{p}+\delta\right) \mu_{l}\left(t_{l}+\epsilon-\gamma-\eta\right)+\mu_{h}\left(t_{h}+\epsilon-\gamma-\eta\right)\right. \\
& \left.+\left(\chi_{l}^{p}+\delta\right) \mu_{l} \kappa_{l}-\left(\chi_{l}^{p}+\delta\right) \mu_{l} \kappa_{l}\right) \\
= & \left(\left(\chi_{l}^{p}+\delta\right) \mu_{l}+\mu_{h}\right)\left[\left(\chi_{l}^{p}+\delta\right) \mu_{l}\left(w_{l}-t_{l}-\epsilon+\gamma+\eta\right)+\mu_{h}\left(w_{h}-t_{h}-\epsilon+\gamma+\eta\right)\right] \\
= & \left(\left(\chi_{l}^{p}+\delta\right) \mu_{l}+\mu_{h}\right)\left[\left(\chi_{l}^{p}+\delta\right) \mu_{l}\left(w_{l}-t_{l}-\epsilon+\gamma+\eta\right)+\mu_{h}\left(w_{h}-t_{h}-\epsilon+\gamma+\eta\right)\right. \\
& \left.-\left(\chi_{l}^{p}+\delta\right) \mu_{l} \kappa_{l}-\mu_{h} \kappa_{h}\right] \\
= & \left(\left(\chi_{l}^{p}+\delta\right) \mu_{l}+\mu_{h}\right)\left[\left(\chi_{l}^{p}+\delta\right) \mu_{l}\left(w_{l}-t_{l}-\epsilon+\gamma+\eta-\kappa_{l}\right)+\mu_{h}\left(w_{h}-t_{h}-\epsilon+\gamma+\eta-\kappa_{h}\right)\right] .
\end{aligned}
$$

Therefore, letting $\hat{\chi}_{l}^{p}=\chi_{l}^{p}+\delta, \hat{\chi}_{l}^{e}=1-\hat{\chi}_{l}^{p}, \hat{\chi}_{l}^{a}=0, \hat{t}_{l}=t_{l}+\epsilon-\gamma-\eta+\kappa_{l}, \hat{t}_{h}=t_{h}+\epsilon-$ $\gamma-\eta+\kappa_{h}$, and $\hat{w}_{e}=\left(\hat{\chi}_{l}^{e} \mu_{l}\right)^{-1}\left(\hat{\chi}_{l}^{p} \mu_{l} \hat{t}_{l}+\mu_{h} \hat{t}_{h}\right)=\left(1-\left(\hat{\chi}_{l}^{p} \mu_{l}+\mu_{h}\right)\right)^{-1}\left(\hat{\chi}_{l}^{p} \mu_{l} \hat{t}_{l}+\mu_{h} \hat{t}_{h}\right)>0$, the regime $\hat{\sigma}=\left(\hat{\chi}_{l}^{p}, \hat{\chi}_{l}^{e}, \hat{\chi}_{l}^{a}, 1,0,0, \hat{t}_{l}, \hat{t}_{h}, \hat{w}_{e}\right)$ implies a balanced budget and satisfies

$$
w_{l}-\hat{t}_{l}=\hat{w}_{e}=\left(\hat{\chi}_{l}^{e} \mu_{l}\right)^{-1}\left(\hat{\chi}_{l}^{p} \mu_{l} \hat{t}_{l}+\mu_{h} \hat{t}_{h}\right)=\left(\hat{\chi}_{l}^{p} \mu_{l}+\mu_{h}\right)\left[\hat{\chi}_{l}^{p} \mu_{l}\left(w_{l}-\hat{t}_{l}\right)+\mu_{h}\left(w_{h}-\hat{t}_{h}\right)\right],
$$

i.e., $\varphi_{l}(\hat{\sigma})=\hat{w}_{e}=\nu(\hat{\sigma})$, as well as $\hat{t}_{l}+\phi \geq \hat{t}_{h}$, because $t_{l}+\phi=t_{h}$ and $\kappa_{l}>0>\kappa_{h}$ so that

$$
t_{l}+\epsilon-\gamma-\eta+\kappa_{l}+\phi \geq t_{h}+\epsilon-\gamma-\eta+\kappa_{h},
$$

and, finally, $\hat{\chi}_{l}^{p}=\chi_{l}^{p}+\delta>0$ and $\hat{\chi}_{l}^{e}=1-\hat{\chi}_{l}^{p}=1-\chi_{l}^{p}-\delta=\chi_{l}^{a}+\chi_{l}^{e}-\delta>\chi_{l}^{a}>0$ as $\chi_{l}^{p}>0$, $\chi_{l}^{e}>0, \chi_{l}^{a}>0$ and $0<\delta<\chi_{l}^{e}$. Thus, $\hat{\sigma}$ satisfies all constraints of Problem $\left(\mathrm{PP}^{\prime}\right)$ but yields a higher objective function value than $\sigma$, because $\hat{\chi}_{l}^{p}=\chi_{l}^{p}+\delta>\chi_{l}^{p}$, a contradiction.

\section{Proposition 6}

Proof. Following Lemma 1, any solution satisfies $\chi_{h}^{p *}=1, \chi_{h}^{e *}=\chi_{h}^{a *}=0$. Following Lemmas 2-3 and Proposition 4, and the details of their proofs, if a solution $\sigma$ satisfies $\chi_{l}^{p}>0$, then it has to solve Problem $\left(\mathrm{PP}^{\prime}\right)$ : the objective function is aggregate output, which is maximized by maximizing $\chi_{l}^{p}$, and for all regimes $\sigma$ with $\chi_{l}^{p}>0$ in the constraint set of Problem (PP), either $\sigma$ or another regime $\hat{\sigma}$ with $\hat{\chi}_{l}^{p}>0$ that is associated with a higher objective function value than $\sigma$ is in the constraint set of Problem $\left(\mathrm{PP}^{\prime}\right)$. Problem $\left(\mathrm{PP}^{\prime}\right)$ has a solution if the 
constraint set is nonempty, because the objective function is continuous and the constraint set is a closed subset of $\Sigma^{\prime}$, which is compact. There are two cases.

Case 1. Suppose there is no regime with $\chi_{l}^{p}>0$ in the constraint set of Problem $\left(\mathrm{PP}^{\prime}\right)$, which includes the case in which the constraint set is empty. By Lemmas 1-3 and Proposition 4, and the details of their proofs, for all regimes $\sigma$ with $\chi_{l}^{p}>0$ in the constraint set of Problem (PP), either $\sigma$ or another regime $\hat{\sigma}$ with $\hat{\chi}_{l}^{p}>0$ that is associated with a higher objective function value than $\sigma$ is in the constraint set of Problem $\left(\mathrm{PP}^{\prime}\right)$. Thus, if there is no regime with $\chi_{l}^{p}>0$ in the latter, then there are no regimes with $\chi_{l}^{p}>0$ in the constraint set of Problem (PP). In this case, as there is no feasible regime with $\chi_{l}^{p}>0$, the solution to Problem (PP) is any regime that has $\chi_{l}^{p *}=0$ and all agents of type $h$ produce, $\chi_{h}^{p *}=1$, and attains an objective function value of $\mu_{h} w_{h}$. The planner cannot do better than the best anarchy regime.

Case 2. Suppose there is a regime $\sigma$ with $\chi_{l}^{p}>0$ in the constraint set of Problem $\left(\mathrm{PP}^{\prime}\right)$. Then, the constraint set is nonempty, Problem $\left(\mathrm{PP}^{\prime}\right)$ has a solution $\sigma^{*}$, and any such solution $\sigma^{*}$ has $\chi_{l}^{p *}>0$, because $\chi_{l}^{p}>0$ is attainable. In addition, following Lemma 4 and Proposition 5 , any such solution $\sigma^{*}$ satisfies $t_{h}^{*}=t_{l}^{*}+\phi$ and $\chi_{l}^{a *}=0$. Thus, $\chi_{l}^{p *}>0, \chi_{l}^{e *}=1-\chi_{l}^{p *}>0$, and $\chi_{l}^{a *}=0$, while $\chi_{h}^{p *}=1$ and $\chi_{h}^{e *}=\chi_{h}^{a *}=0$, so that there are no appropriators and, as $\theta=q$ and $(1-\theta)=(1-q)$, all $(1-q)$ agents that do not produce are employed in enforcement. Combining these insights with constraints (21)-(26), the following holds: If there is a regime $\sigma$ with $\chi_{l}^{p}>0$ in the constraint set of Problem $\left(\mathrm{PP}^{\prime}\right)$, then a solution $\sigma^{*}$ to Problem $\left(\mathrm{PP}^{\prime}\right)$ exists and satisfies both $\chi_{l}^{p *}>0$ and the system of equations (39)-(43), where

$$
\begin{aligned}
& t_{h}=t_{l}+\phi, \\
& w_{l}-t_{l}=w_{e}, \\
& (1-\theta) w_{e}=\left(\chi_{l}^{p} \mu_{l} t_{l}+\mu_{h} t_{h}\right), \\
& w_{e}=\theta\left[\chi_{l}^{p} \mu_{l}\left(w_{l}-t_{l}\right)+\mu_{h}\left(w_{h}-t_{h}\right)\right], \\
& \theta=\chi_{l}^{p} \mu_{l}+\mu_{h} .
\end{aligned}
$$

Equations (39)-(43) are five equations in five unknowns $\chi_{l}^{p}, t_{l}, t_{h}, w_{e}$, and $\theta$. For a solution to this system to be consistent with $\chi_{l}^{p *}>0$, it has to satisfy $\theta=\chi_{l}^{p} \mu_{l}+\mu_{h}>\mu_{h}$. Using (39), (40), and (43) to replace $t_{h}, w_{e}$, and $\left(\chi_{l}^{p} \mu_{l}+\mu_{h}\right)$ in (41) and (42) gives

$$
\begin{aligned}
& (1-\theta)\left(w_{l}-t_{l}\right)=\theta t_{l}+\mu_{h} \phi, \\
& w_{l}-t_{l}=\theta\left[\chi_{l}^{p} \mu_{l} w_{l}+\mu_{h} w_{h}\right]-\theta^{2} t_{l}-\theta \mu_{h} \phi .
\end{aligned}
$$

Rewriting (44) yields

$$
t_{l}=(1-\theta) w_{l}-\mu_{h} \phi
$$


which using (40) gives

$$
w_{e}=\theta w_{l}+\mu_{h} \phi
$$

both as functions of parameters and $\theta$. Plugging $t_{l}$ into (45) gives

$$
\begin{aligned}
& w_{l}-(1-\theta) w_{l}+\mu_{h} \phi=\theta\left[\chi_{l}^{p} \mu_{l} w_{l}+\mu_{h} w_{h}\right]-\theta^{2}\left((1-\theta) w_{l}-\mu_{h} \phi\right)-\theta \mu_{h} \phi \\
\Leftrightarrow & \theta w_{l}+\mu_{h} \phi=\theta\left[\chi_{l}^{p} \mu_{l} w_{l}+\mu_{h} w_{h}\right]-\theta^{2} w_{l}+\theta^{2} \theta w_{l}+\theta^{2} \mu_{h} \phi-\theta \mu_{h} \phi \\
\Leftrightarrow & \left(1-\theta^{2}\right)\left(\theta w_{l}+\mu_{h} \phi\right)=\theta\left[\chi_{l}^{p} \mu_{l} w_{l}+\mu_{h} w_{h}-\theta w_{l}-\mu_{h} \phi\right] \\
\Leftrightarrow & \left(1-\theta^{2}\right)\left(\theta w_{l}+\mu_{h} \phi\right)=\theta\left[\chi_{l}^{p} \mu_{l} w_{l}+\mu_{h} w_{h}-\left(\chi_{l}^{p} \mu_{l}+\mu_{h}\right) w_{l}-\mu_{h} \phi\right] \\
\Leftrightarrow & \left(1-\theta^{2}\right)\left(\theta w_{l}+\mu_{h} \phi\right)=\theta \mu_{h}\left(w_{h}-w_{l}-\phi\right),
\end{aligned}
$$

i.e., one equation in one unknown, $\theta$. Thus, suppressing parameters, let $h:(0,1) \rightarrow \mathbb{R}$ be

$$
h(\theta)=\theta \mu_{h}\left(w_{h}-w_{l}-\phi\right)-\left(1-\theta^{2}\right)\left(\theta w_{l}+\mu_{h} \phi\right) .
$$

For all $\phi \in\left(0,\left(w_{h}-w_{l}\right)\right), h$ is a strictly convex function of $\theta$ with

$$
\lim _{\theta \rightarrow 0} h(\theta)=-\mu_{h} \phi<0 \text { and } \quad \lim _{\theta \rightarrow 1} h(\theta)=\mu_{h}\left(w_{h}-w_{l}-\phi\right)>0,
$$

and thus has a unique root $\theta^{*} \in(0,1)$ such that $h\left(\theta^{*}\right)=0$. An increase in $\phi$ decreases $h(\theta)$ for all $\theta$, and thus increases $\theta^{*}$. Via (43), (46), (39), and (47), the unique $\theta^{*}$ implies unique

$$
\begin{aligned}
& \chi_{l}^{p *}=\mu_{l}^{-1}\left(\theta^{*}-\mu_{h}\right), \\
& t_{l}^{*}=\left(1-\theta^{*}\right) w_{l}-\mu_{h} \phi, \\
& t_{h}^{*}=t_{l}^{*}+\phi, \\
& w_{e}^{*}=\theta^{*} w_{l}+\mu_{h} \phi,
\end{aligned}
$$

completing the unique solution to system (39)-(43). As $\theta^{*} \in(0,1)$ and $\phi \in\left(0,\left(w_{h}-w_{l}\right)\right)$, this unique solution satisfies $\chi_{l}^{p *}<1, t_{l}^{*} \in\left[-w_{h}, w_{l}\right], t_{h}^{*} \in\left[-w_{h}, w_{h}\right]$, and $w_{e}^{*} \in\left[0, w_{h}\right]$. It is consistent with $\chi_{l}^{p *}>0$ if and only if $\theta^{*}>\mu_{h}$. If $\theta^{*} \leq \mu_{h}$, then the unique solution to (39)(43) does not satisfy $\chi_{l}^{p *}>0$. Then, by the statement in italics above, there is no regime with $\chi_{l}^{p}>0$ in the constraint set of Problem $\left(\mathrm{PP}^{\prime}\right)$, and case 1 applies. The condition $\theta^{*}>\mu_{h}$ is satisfied and case 2 applies (is violated and case 1 applies) if, plugging $\mu_{h}$ into $h$,

$$
\begin{aligned}
& h\left(\mu_{h}\right)=\mu_{h}^{2}\left(w_{h}-w_{l}-\phi\right)-\left(1-\mu_{h}^{2}\right)\left(\mu_{h} w_{l}+\mu_{h} \phi\right)<(\geq) 0 \\
& \Leftrightarrow \quad \mu_{h}\left(w_{h}-w_{l}-\phi\right)<(\geq)\left(1-\mu_{h}^{2}\right)\left(w_{l}+\phi\right) \\
& \Leftrightarrow \quad\left(1+\mu_{h}\left(1-\mu_{h}\right)\right) \phi>(\leq) \mu_{h} w_{h}-\left(1+\mu_{h}\left(1-\mu_{h}\right)\right) w_{l}
\end{aligned}
$$




$$
\begin{aligned}
\Leftrightarrow \quad\left(1+\mu_{h} \mu_{l}\right) \phi & >(\leq) \mu_{h} w_{h}-\left(1+\mu_{h} \mu_{l}\right) w_{l} \\
\Leftrightarrow \quad \phi & >(\leq) \underline{\phi} \equiv \frac{\mu_{h} w_{h}-\left(1+\mu_{l} \mu_{h}\right) w_{l}}{\left(1+\mu_{l} \mu_{h}\right)}
\end{aligned}
$$

which is the stated condition. If $\phi>\underline{\phi}$, combining the unique solution to system (39)-(43) with $\chi_{l}^{e *}=1-\chi_{l}^{p *}>0, \chi_{l}^{a *}=0, \chi_{h}^{p *}=1$, and $\chi_{h}^{e *}=\chi_{h}^{a *}=0$ gives the unique solution $\sigma^{*}$. Finally, suppose $\phi>\underline{\phi}$. Then, from (50), $t_{l}^{*}=\left(1-\theta^{*}\right) w_{l}-\mu_{h} \phi$. From the definition of $h$ in (48), with $\phi \rightarrow\left(w_{h}-w_{l}\right), h(\theta) \rightarrow-\left(1-\theta^{2}\right)\left(\theta w_{l}+\mu_{h}\left(w_{h}-w_{l}\right)\right)<0$ for all $\theta<1$, so that $\theta^{*} \rightarrow 1$ with $\phi \rightarrow\left(w_{h}-w_{l}\right)$ : for all $\epsilon>0, h(1-\epsilon)<0$ for some $\phi \in\left(0,\left(w_{h}-w_{l}\right)\right)$, $\phi$ close enough to $\left(w_{h}-w_{l}\right)$, so that $\theta^{*}>1-\epsilon$. Thus, $t_{l}^{*} \rightarrow-\mu_{h}\left(w_{h}-w_{l}\right)<0$ with $\phi \rightarrow\left(w_{h}-w_{l}\right)$. It follows that for high enough $\phi, t_{l}^{*}<0$, which completes the proof.

\section{Proposition 7}

Proof. The function $h$ as defined in equation (48) is a strictly convex function of $\theta$, negativevalued for $\theta$ approaching zero and switching sign with $\theta$ approaching one, therefore has a positive derivative at its unique root $\theta^{*}$, and a higher $\phi$ decreases it for all $\theta$. Hence, $\theta^{*}$ increases with $\phi$. As $\phi>\underline{\phi},(49)-(52)$ apply. From (49), $\chi_{l}^{p *}$ increases, so that $\chi_{l}^{e *}=1-\chi_{l}^{p *}$ decreases; from (52), $w_{e}^{*}$ increases, as it increases in both $\theta^{*}$ and $\phi$; from (50), $t_{l}^{*}$ decreases, as it decreases in both $\theta^{*}$ and $\phi$; and from (51) the difference $t_{h}^{*}-t_{l}^{*}=\phi$ increases with $\phi$.

\section{References}

Acemoglu, D. (1995). Reward Structures and the Allocation of Talent. European Economic Review 39(1), 17-33.

Acemoglu, D. (2005). Politics and Economics in Weak and Strong States. Journal of Monetary Economics 52(7), 1199-1226.

Acemoglu, D. and S. Johnson (2005). Unbundling Institutions. Journal of Political Economy 113(5), 949-995.

Acemoglu, D. and T. Verdier (1998). Property Rights, Corruption and the Allocation of Talent: A General Equilibrium Approach. The Economic Journal 108, 1381-1403.

Acemoglu, D. and T. Verdier (2000). The Choice between Market Failures and Corruption. The American Economic Review 90, 194-211.

Allingham, M. G. and A. Sandmo (1972). Income Tax Evasion: A Theoretical Analysis. Journal of Public Economics 1, 323-338.

Andreoni, J., B. Erard, and J. Feinstein (1998). Tax Compliance. Journal of Economic Literature 36(2), 818-860. 
Basu, K., S. Bhattacharya, and A. Mishra (1992). Notes on Bribery and the Control of Corruption. Journal of Public Economics 48(3), 349-359.

Baumol, W. J. (1990). Entrepreneurship: Productive, Unproductive, and Destructive. The Journal of Political Economy 98(5), 893-921.

Becker, G. S. (1968). Crime and Punishment: An Economic Approach. Journal of Political Economy 76(2), 169-217.

Becker, G. S. and G. J. Stigler (1974). Law Enforcement, Malfeasance and Compensation of Enforcers. The Journal of Legal Studies 3(1), 1-18.

Benoît, J.-P. and M. J. Osborne (1995). Crime, Punishment and Social Expenditure. Journal of Institutional and Theoretical Economics 151(2), 326-347.

Besley, T. and J. McLaren (1993). Taxes and Bribery: The Role of Wage Incentives. The Economic Journal 103(416), 119-141.

Besley, T. and T. Persson (2009). The Origins of State Capacity: Property Rights, Taxation, and Politics. The American Economic Review 99(4), 1218-1244.

Besley, T. and T. Persson (2010). State Capacity, Conflict, and Development. Econometrica 78(1), 1-34.

Casamatta, G. (2011). Optimal Income Taxation with Tax Avoidance. Working Paper.

Chander, P. and L. Wilde (1992). Corruption in Tax Administration. Journal of Public Economics 49(3), 333-349.

Ehrlich, I. (1973). Participation in Illegitimate Activities: A Theoretical and Empirical Investigation. Journal of Political Economy 81(3), 521-565.

Grochulski, B. (2007). Optimal Nonlinear Income Taxation with Costly Tax Avoidance. Federal Reserve Bank of Richmond Economic Quarterly 93(1), 77-109.

Hall, R. E. and C. I. Jones (1999). Why Do Some Countries Produce So Much More Output Per Worker Than Others? The Quarterly Journal of Economics 114(1), 83-116.

Herrera, H. and C. Martinelli (2013). Oligarchy, Democracy, and State Capacity. Economic Theory 52, 165-186.

Hindriks, J., M. Keen, and A. Muthoo (1999). Corruption, Extortion and Evasion. Journal of Public Economics 74, 395-430.

İmrohoroğlu, A., A. Merlo, and P. Rupert (2000). On the Political Economy of Income Redistribution and Crime. International Economic Review 41(1), 1-26.

Internal Revenue Service (2016). Federal Tax Compliance Research: Tax Gap Estimates for Tax Years 2008-2010. Research, Analysis and Statistics, Publication 1415 (Rev. 5-2016), Washington, DC, May 2016. 
Knack, S. and P. Keefer (1995). Institutions and Economic Performance: Cross-Country Tests Using Alternative Institutional Measures. Economics and Politics 7(3), 207-227.

Koeppl, T., C. Monnet, and E. Quintin (2014). Efficient Contract Enforcement. Economic Theory 55, 161-183.

Konrad, K. A. and S. Skaperdas (2012). The Market for Protection and the Origin of the State. Economic Theory 50, 417-443.

Lacker, J. M. and J. A. Weinberg (1989). Optimal Contracts under Costly State Falsification. The Journal of Political Economy 97(6), 1345-1363.

Mookherjee, D. and I. P. L. Png (1995). Corruptible law enforcers: How should they be compensated. The Economic Journal 105, 145-159.

Moselle, B. and B. Polak (2001). A Model of a Predatory State. The Journal of Law, Economics, and Organization 17(1), 1-33.

Murphy, K. M., A. Shleifer, and R. W. Vishny (1991). The Allocation of Talent: Implications for Growth. The Quarterly Journal of Economics 106(2), 503-530.

Murphy, K. M., A. Shleifer, and R. W. Vishny (1993). Why Is Rent-Seeking So Costly to Growth? The American Economic Review, Papers and Proceedings 83(2), 409-414.

Olson, M. (1993). Dictatorship, Democracy, and Development. American Political Science Review 87(3), 567-576.

Piketty, T., E. Saez, and G. Zucman (2018). Distributional National Accounts: Methods and Estimates for the United States. The Quarterly Journal of Economics 133(2), 553-609.

Polinsky, A. M. and S. Shavell (2001). Corruption and Optimal Law Enforcement. Journal of Public Economics 81(1), 1-24.

Quintin, E. (2008). Contract Enforcement and the Size of the Informal Economy. Economic Theory 37(3), 395-416.

Reinganum, J. F. and L. L. Wilde (1985). Income Tax Compliance in a Principal-Agent Framework. Journal of Public Economics 26, 1-18.

Rose-Ackerman, S. (1975). The Economics of Corruption. Journal of Public Economics 4(2), $187-203$.

Schneider, F. and D. H. Enste (2000). Shadow Economies: Size, Causes, and Consequences. Journal of Economic Literature 38(1), 77-114.

Stigler, G. J. (1970). The Optimum Enforcement of Laws. Journal of Political Economy 78(3), $526-536$.

Tullock, G. (1967). The Welfare Costs of Tariffs, Monopolies, and Theft. Western Economic Journal 5(3), 224-232. 